\title{
FINITE ELEMENT APPROXIMATION \\ TO INITIAL-BOUNDARY VALUE PROBLEMS \\ OF THE SEMICONDUCTOR DEVICE EQUATIONS WITH MAGNETIC INFLUENCE
}

\author{
JIANG ZHU
}

\begin{abstract}
We shall consider Zlámal's approach to the nonstationary equations of the semiconductor device theory under magnetic fields, with mixed boundary conditions. Owing to the reduced smoothness of the electric potential $\psi$ and carrier densities $n$ and $p$ caused by considering the mixed boundary conditions, we must use a nonstandard analysis for this procedure. Existence as well as uniqueness of the approximate solution is proved. The convergence rates obtained in this paper are slower than those previously obtained for pure Dirichlet or Neumann boundary conditions.
\end{abstract}

\section{INTRODUCTION}

We shall consider a system of three quasilinear partial differential equations in a bounded polygonal domain $\Omega \in \mathbf{R}^{2}$, which form a basic model of the transient behavior of a semiconductor device in a magnetic field (cf. Allegretto, Mun, Nathan, and Baltes [1], and Wang [30]):

$$
\begin{aligned}
& \text { (a) }-\Delta \psi=\frac{q}{\varepsilon}(p-n+N), \\
& \text { (b) } \frac{\partial n}{\partial t}-\nabla \cdot\left[e^{a \psi} A_{1} \nabla\left(e^{-a \psi} n\right)\right]+R_{n}(n, p)=0, \\
& \text { (c) } \frac{\partial p}{\partial t}-\nabla \cdot\left[e^{-a \psi} A_{2} \nabla\left(e^{a \psi} p\right)\right]+R_{p}(n, p)=0 .
\end{aligned}
$$

The unknowns are the electrostatic potential $\psi$ and the electron and hole densities $n$ and $p$, while $q$ and $\varepsilon$ are constants ( $q$ is the electron charge, $\varepsilon$ is the permittivity). The function $N$ is the total electric active net impurity density or "doping", and $a$ is a positive constant; $R_{n}(n, p)$ and $R_{p}(n, p)$ are the recombination rates. The matrices

$$
A_{i}=\left(\begin{array}{ll}
a_{11}^{(i)} & a_{12}^{(i)} \\
a_{21}^{(i)} & a_{22}^{(i)}
\end{array}\right)=\frac{1}{1+\beta^{2}}\left(\begin{array}{cc}
1 & (-1)^{i} \beta \\
(-1)^{i-1} \beta & 1
\end{array}\right), \quad i=1,2,
$$

Received by the editor June 29, 1990 and, in revised form, April 30, 1991.

1991 Mathematics Subject Classification. Primary 65N30.

Key words and phrases. Finite element approximation, semiconductor device equation, magnetic field.

(C) 1992 American Mathematical Society $0025-5718 / 92 \$ 1.00+\$ .25$ per page 
are positive definite nonsymmetric. The reason for the asymmetry of $A_{i} \quad(i=$ $1,2)$ is the presence of a magnetic field $\vec{B}=(0,0, \beta)$, where $\beta=\beta(x)$ is Lipschitz continuous. Indeed, if $\vec{B}=0$, then $A_{i} \quad(i=1,2)$ are positive definite symmetric. Since $A_{i}(i=1,2)$ are positive definite and bounded, there are two positive constants $a_{0}$ and $M$ such that, for any $u=\left(u_{1}, u_{2}\right)$, $v=\left(v_{1}, v_{2}\right) \in \mathbf{R}^{2}$,

$$
\begin{aligned}
a_{0}|u|^{2} \leq\left(A_{i} u, u\right), & i=1,2, \\
\left|\left(A_{i} u, v\right)\right| \leq M|u||v|, & i=1,2,
\end{aligned}
$$

with $|u|^{2}=\sum_{i=1}^{2} u_{i}^{2}$.

We simplify the system, taking $a=q / \varepsilon=1$ and $R_{n}(n, p)=R_{p}(n, p)=$ $R(n, p)$ (assumed Lipschitz continuous). These simplifications are not essential, neither for the construction of the approximate solution nor for the results of this paper. We can write (1.1) in the form

$$
\begin{aligned}
& \text { (a) }-\Delta \psi=p-n N, \\
& \text { (b) } \frac{\partial n}{\partial t}-\nabla \cdot\left[A_{1}(\nabla n-n \nabla \psi)\right]+R(n, p)=0, \\
& \text { (c) } \frac{\partial p}{\partial t}-\nabla \cdot\left[A_{2}(\nabla p+p \nabla \psi)\right]+R(n, p)=0 .
\end{aligned}
$$

For simplicity, consider the PDE system (1.5) with homogeneous mixed boundary conditions:

$$
\begin{gathered}
\{\psi, n, p\}=\{0,0,0\} \quad \text { on } \partial \Omega_{D} \times I, \\
\left\{\frac{\partial \psi}{\partial \nu}, J_{n} \cdot \nu, J_{p} \cdot \nu\right\}=\{0,0,0\} \quad \text { on } \partial \Omega_{N} \times I .
\end{gathered}
$$

Here, the boundary $\partial \Omega$ of $\Omega$ has been decomposed into the union $\partial \Omega_{D} \cup \partial \Omega_{N}$, where $\partial \Omega_{D}$ is of positive measure in $\partial \Omega, I=[0, T], \nu$ is the outward unit normal vector on $\partial \Omega, J_{n}=A_{1}(\nabla n-n \nabla \psi)$, and $J_{p}=A_{2}(\nabla p+p \nabla \psi)$.

In addition, we have the initial condition

$$
n=n^{0}(x), \quad p=p^{0}(x) \quad \text { in } \Omega .
$$

Remark 1.1. For nonhomogeneous mixed boundary conditions with smooth data, the problems can be homogenized by Banasiak and Roach's trace theorem in [2].

There is much work concerning the basic semiconductor device equations with no magnetic fields. For stationary problems, Mock [23, 25] showed the existence and uniqueness of a solution subject to the mixed boundary conditions (with $R=0$ ). A very similar existence proof was given by Bank, Jerome, and Rose [4], and effective numerical algorithms were also presented in their paper. Later, Jerome [17] proved the existence for a more general stationary problem. A singular perturbation analysis for the problems was given by Markowich [19, 20], Markowich and Ringhofer [21], and Selberherr and Ringhofer [27]. Finite difference or finite element methods are discussed in Markowich [20] and in references therein. Recently, Ringhofer and Schmeiser [26] analyzed an iterative method and its convergence. For nonstationary problems, Mock [24] was the first to prove a global existence and uniqueness result, and a more general type 
of the boundary conditions was discussed in Gajewski [13] and Gajewski and Gröger [14]. With regard to numerical treatments, Zlámal [33] has proposed two fully discrete finite element schemes (one is nonlinear, the other is partly linear) and discussed the existence (for both schemes) and uniqueness (for the second scheme) of the approximations. Stability, uniqueness, and convergence (for the first scheme) of the approximations have been investigated under stronger assumptions in Zlámal [34]. The mixed finite element-characteristic procedure for the one-dimensional Dirichlet problem was introduced by Douglas, Gamba, and Squeff [7], and Gamba and Squeff [15], Douglas and Yuan [8], and Douglas, Yuan, and Li [9, 10] have discussed, respectively, the finite differencecharacteristic finite difference procedure, the mixed finite element-characteristic finite difference procedure, and the mixed finite element-characteristic finite element procedure for the two-dimensional Dirichlet problem and the Neumann problem, and have given the convergence analyses under the assumption of a smooth solution.

However, to our knowledge, there is not much work on problems in the presence of magnetic fields. A finite element analysis for stationary problems was given by Allegretto, Mun, Nathan, and Baltes [1], but there was no theoretical analysis of approximation in their paper. Recently, the author [32] presented and analyzed the problem (1.1) by the finite difference-characteristic finite difference procedure, considering nonhomogeneous Dirichlet boundary conditions.

Unfortunately, it is well known (see, e.g., $[2,28]$ ) that in general the solutions of mixed boundary value problems for elliptic equations are not smooth, no matter how smooth the data may be, and moreover, the loss of smoothness occurs in the vicinity of $\overline{\partial \Omega}_{D} \cap \overline{\partial \Omega}_{N}$. Hence, the solutions of (1.5)-(1.8) are certainly not smooth. Similar to the idea of Ewing and Wheeler [12], we shall use in this paper a nonstandard analysis for Zlámal's approach. Since the resulting functions are considerably less smooth than previously assumed, the convergence rates obtained in this paper are slower than those previously obtained. Recently, Markowich and Zlámal [22] have generalized Zlámal's approach to mixed boundary value problems of second-order elliptic equations.

The paper contains two additional sections. In $\S 2$, terminology is developed, a variational form of the problem (1.5)-(1.8), basic regularity and boundedness assumptions are presented, and the continuous-time Zlamal's approach to (1.5)(1.8) is defined. In $\S 3$, existence, uniqueness, and a priori error estimates for this approach are obtained. Throughout, the symbols $C$ and $\delta$ will denote, respectively, a generic constant and a generic small positive constant.

\section{PRELIMINARIES AND DESCRIPTION OF APPROXIMATIONS}

Let $(u, v)=\int_{\Omega} u v d x$ and $\|u\|^{2}=(u, u)$ be the standard $L^{2}$ inner product and norm. Let $W_{q}^{k}(\Omega)$ be the Sobolev space on $\Omega$ with norm

$$
\|u\|_{W_{q}^{k}}=\left(\sum_{|\alpha| \leq k}\left\|\frac{\partial^{\alpha} u}{\partial x^{\alpha}}\right\|_{L^{q}(\Omega)}^{q}\right)^{1 / q},
$$

with the usual modification for $q=\infty$. If $U=\left(u_{1}, u_{2}\right)$, write $\|U\|_{W_{q}^{k}}$ in place of $\left(\left\|u_{1}\right\|_{W_{q}^{k}}^{q}+\left\|u_{2}\right\|_{W_{q}^{k}}^{q}\right)^{1 / q}$. When $q=2$, denote $\|u\|_{W_{q}^{k}}=\|u\|_{H^{k}}=\|u\|_{k}$. 
If $k=0,\|u\|_{0}=\|u\|$. We also denote by $H^{1+\sigma}(\Omega) \quad(\sigma$ is a real number with $0<\sigma<1$ ) the noninteger Sobolev space on $\Omega$ with norm (see, e.g., Girault and Raviart [16])

$$
\begin{aligned}
\|u\|_{1+\sigma} & =\|u\|_{H^{1+\sigma}(\Omega)} \\
& =\left\{\|u\|_{1}^{2}+\sum_{|\alpha|=1} \int_{\Omega} \int_{\Omega} \frac{\left|\partial^{\alpha} u(x)-\partial^{\alpha} u(y)\right|^{2}}{|x-y|^{2(1+\sigma)}} d x d y\right\}^{1 / 2},
\end{aligned}
$$

where $|x|$ denotes the Euclidean norm of $\mathbf{R}^{2}$. Let $I=[0, T]$, and

$$
V=\left\{v \in H^{1}(\Omega) ;\left.v\right|_{\partial \Omega_{D}}=0\right\} .
$$

Multiplying (1.5) by a function $v \in V$, integrating over $\Omega$, and using Green's theorem and (1.7), we have
(a) $d(\psi, v)=(p-n+N, v), \quad v \in V$,
(b) $\left(\frac{\partial n}{\partial t}, v\right)+\nu(\psi ; n, v)+(R(n, p), v)=0, \quad v \in V$,
(c) $\left(\frac{\partial p}{\partial t}, v\right)+\pi(\psi ; p, v)+(R(n, p), v)=0, \quad v \in V$,

where

$$
\begin{aligned}
d(\psi, v) & =\int_{\Omega} \nabla \psi \cdot \nabla v d x \\
\nu(\psi ; n, v) & =\int_{\Omega} A_{1}(\nabla n-n \nabla \psi) \cdot \nabla v d x \\
\pi(\psi ; p, v) & =\int_{\Omega} A_{2}(\nabla p+p \nabla \psi) \cdot \nabla v d x .
\end{aligned}
$$

We are looking for $\{\psi, n, p\}: I \rightarrow V \times V \times V$.

Let $\{\psi, n, p\}$, the solution of $(1.5)-(1.8)$, satisfy the following regularity assumptions:

(a) $\|\psi\|_{L^{\infty}\left(H^{1+\sigma}\right)}+\|n\|_{L^{2}\left(H^{1+\sigma}\right)}+\|p\|_{L^{2}\left(H^{1+\sigma}\right)} \leq C$,

(b) $\|n\|_{L^{\infty}\left(L^{\infty}\right)}+\|p\|_{L^{\infty}\left(L^{\infty}\right)} \leq C$,

(c) $\left\|\frac{\partial n}{\partial t}\right\|_{L^{2}\left(L^{2}\right)}+\left\|\frac{\partial p}{\partial t}\right\|_{L^{2}\left(L^{2}\right)} \leq C$,

where $0<\sigma<1$ and $C$ are fixed constants, $\|u\|_{L^{q}(X)}=\|u\|_{L^{q}(I ; X)}, q=2, \infty$, and $X$ is a Sobolev space on $\Omega$. In view of $[1,2,4,13,14,28]$, the above assumptions are reasonable.

In this paper we restrict our attention to continuous-time Zlámal's approach (nonlinear scheme) to (1.5)-(1.8). We consider a family $\left\{T_{h}\right\}$ of triangulations of $\bar{\Omega}$. Let $K$ denote an element of $T_{h}, h_{K}=\operatorname{diam}(K)$, and $h=\max _{K \in T_{h}} h_{K}$. As in Zlámal [34], we assume that the family $\left\{T_{h}\right\}$ satisfies the minimum angle condition and is of acute type. Thus, if $J$ is the Jacobian matrix of the linear 
mapping which maps a given triangle $K$ on the reference triangle $\widehat{K}$, then

$$
C h^{2} \leq|\operatorname{det} J| \leq C^{-1} h^{2} \quad \forall K \in \bigcup_{h} T_{h} .
$$

We shall use the above implicitly in some places.

With each partition from $\left\{T_{h}\right\}$ we associate the finite-dimensional space $V_{h}=\left\{v_{h} \in C(\bar{\Omega}) ; v_{h}\right.$ is a linear polynomial on each $\left.K \in T_{h},\left.v_{h}\right|_{\partial \Omega_{D}}=0\right\}$.

We use the same idea as in Mock [25] and Zlámal [33, 34]: the quantities $J_{n}, J_{p}$, and $\|\nabla \psi\|$ are approximated by constants on each element $K$. Let $\psi_{h}, n_{h}, p_{h}$, and $v_{h}$ belong to $V_{h}$. The discrete analogs of the forms $\nu(\psi ; n, v)$ and $\pi(\psi ; p, v)$ are

$$
\begin{array}{r}
\nu_{h}\left(\psi_{h} ; n_{h}, v_{h}\right)=\sum_{K \in T_{h}} \sum_{r=j, k, m} v_{h r}\left\{\int_{K} A_{1}^{K}\left(J^{T}\right)^{-1} D^{K} J^{T} \nabla n_{h} \cdot \nabla v^{r} d x\right. \\
\left.-\int_{K} n_{h r} A_{1}^{K} \nabla \psi_{h} \cdot \nabla v^{r} d x\right\}, \\
\pi_{h}\left(\psi_{h} ; p_{h}, v_{h}\right)=\sum_{K \in T_{h}} \sum_{r=j, k, m} v_{h r}\left\{\int_{K} A_{2}^{K}\left(J^{T}\right)^{-1} B^{K} J^{T} \nabla p_{h} \cdot \nabla v^{r} d x\right. \\
\left.+\int_{K} p_{h r} A_{2}^{K} \nabla \psi_{h} \cdot \nabla v^{r} d x\right\},
\end{array}
$$

where

$$
A_{i}^{K}=\left(\begin{array}{cc}
a^{K} & (-1)^{i} b^{K} \\
(-1)^{i-1} b^{K} & a^{K}
\end{array}\right), \quad i=1,2,
$$

$a^{K}=1 /\left(1+\left(\beta^{K}\right)^{2}\right), b^{K}=\beta^{K} /\left(1+\left(\beta^{K}\right)^{2}\right), \beta^{K}=\beta\left(x^{K}\right)$, and $x^{K}$ is the center of gravity of the element $K$. Here, $J$ is the Jacobian matrix of the mapping which maps $K$ on $\widehat{K}$ in such a way that the node $x^{r}$ is mapped on the vertex $(0,0)$ in the reference plane (see Zlámal [33]), $v_{h r}$ is the value $v_{h}\left(x^{r}\right), v^{r}$ is the basis function associated with the node $x^{r}$, and $B^{K}, D^{K}$ are the matrices

$$
\begin{aligned}
& B^{K}=\operatorname{diag}\left(B\left(\psi_{h 1}-\psi_{h 2}\right), B\left(\psi_{h 1}-\psi_{h 3}\right)\right), \\
& D^{K}=\operatorname{diag}\left(D\left(\psi_{h 1}-\psi_{h 2}\right), D\left(\psi_{h 1}-\psi_{h 3}\right)\right) .
\end{aligned}
$$

Here, $B(\zeta)=\zeta\left(e^{\zeta}-1\right)^{-1}$ and $D(\zeta)=e^{\zeta} B(\zeta)=B(-\zeta),-\infty<\zeta<\infty$. Furthermore, $\psi_{h 1}, \psi_{h 2}, \psi_{h 3}$ are the local notations of the values of $\psi_{h}$ at the vertices $x^{j}, x^{k}, x^{m}$ such that $\psi_{h 1}=\psi_{h r}, r=j, k, m$.

Remark 2.1. For $A_{i}^{K}, i=1,2$, the inequalities (1.3) and (1.4) are still valid.

The $L^{2}(\Omega)$-scalar product $(\cdot, \cdot)$ will be approximated by $(\cdot, \cdot)_{h}$ defined in Zlámal [33] $\left((u, v)_{h}=\sum_{j=1}^{q} m_{j} u_{j} v_{j}, m_{j}>0, q\right.$ is the number of all nodes not lying on $\left.\partial \Omega_{D}\right)$. Let $f_{I}$ denote the interpolate of a given function $f$.

Now we can introduce the continuous-time approximation of $\{\psi, n, p\}$ as follows: let $\left\{\psi_{h}, n_{h}, p_{h}\right\}: I \rightarrow V_{h} \times V_{h} \times V_{h}$ be defined by 
(a) $d\left(\psi_{h}, v_{h}\right)=\left(p_{h}-n_{h}+N_{I}, v_{h}\right)_{h}, \quad v_{h} \in V_{h}$,

(b) $\left(\frac{\partial n_{h}}{\partial t}, v_{h}\right)_{h}+\nu_{h}\left(\psi_{h} ; n_{h}, v_{h}\right)+\left(R\left(n_{h}, p_{h}\right), v_{h}\right)_{h}=0, \quad v_{h} \in V_{h}$,

(c) $\left(\frac{\partial p_{h}}{\partial t}, v_{h}\right)_{h}+\pi_{h}\left(\psi_{h} ; p_{h}, v_{h}\right)+\left(R\left(n_{h}, p_{h}\right), v_{h}\right)_{h}=0, \quad v_{h} \in V_{h}$,

(d) $n_{h}(0)=n_{I}^{0}, \quad p_{h}(0)=p_{I}^{0}$.

The main results of this paper are the existence, uniqueness, and a priori error estimates for the approximation $\left\{\psi_{h}, n_{h}, p_{h}\right\}$. These will be developed in the next section.

\section{EXISTENCE, UNIQUENESS, AND A PRIORI ERROR ESTIMATES}

Similar to the idea introduced by Ewing and Wheeler [12] for miscible displacement problems, we first define the $L^{2}$ projection $\{\bar{n}, \bar{p}\}$ of $\{n, p\}$ into $V_{h} \times V_{h}$ by
(a) $\left(n-\bar{n}, v_{h}\right)=0, \quad v_{h} \in V_{h}$, or
(b) $\left(\frac{\partial n}{\partial t}-\frac{\partial \bar{n}}{\partial t}, v_{h}\right)=0, \quad v_{h} \in V_{h}$;
(c) $\left(p-\bar{p}, v_{h}\right)=0, \quad v_{h} \in V_{h}$, or
(d) $\left(\frac{\partial p}{\partial t}-\frac{\partial \bar{p}}{\partial t}, v_{h}\right)=0, \quad v_{h} \in V_{h}$.

We are led to use the $L^{2}$ projection of $\{n, p\}$ into $V_{h} \times V_{h}$ instead of the now more standard $H^{1}$ projection, owing to smoothness restrictions on $n$ and $p$. Since we assume that $\frac{\partial n}{\partial t}$ and $\frac{\partial p}{\partial t}$ are only in $L^{2}\left(I ; L^{2}\right)$, we are not able to treat terms like $\frac{\partial}{\partial t}(n-\bar{n})$ and $\frac{\partial}{\partial t}(p-\bar{p})$ in the usual fashion. Thus, we have used $\{\bar{n}, \bar{p}\}$ in $(3.1 \mathrm{~b}, \mathrm{~d})$ to remove this problem. Using the theory of interpolation spaces, we obtain (see, e.g., Ewing and Wheeler [12])

Lemma 3.1. There exists a positive constant $C$ such that, for each $t \in I$,
(a) $\|n-\bar{n}\|+h\|n-\bar{n}\|_{1} \leq C\|n\|_{s_{1}} h^{s_{1}}$
$1 \leq s_{1} \leq 1+\sigma$,
(b) $\|p-\bar{p}\|+h\|p-\bar{p}\|_{1} \leq C\|p\|_{s_{1}} h^{s_{1}}$,
$1 \leq s_{1} \leq 1+\sigma$,
(c) $\|n-\bar{n}\|_{L^{\infty}} \leq C\|n\|_{W_{\infty}^{s_{2}}} h^{s_{2}}, \quad 0 \leq s_{2} \leq 1$,
(d) $\|p-\bar{p}\|_{L^{\infty}} \leq C\|p\|_{W_{\infty}^{s_{2}}} h^{s_{2}}, \quad 0 \leq s_{2} \leq 1$,
(e) $\left\|v-v_{I}\right\|+h\left\|v-v_{I}\right\|_{1} \leq C\|v\|_{s_{3}} h^{s_{3}} \quad \forall v \in H^{s_{3}}(\Omega), \quad 1 \leq s_{3} \leq 1+\sigma$.

Assuming that the family $\left\{V_{h}\right\}$ satisfies the following inverse inequalities (see Ciarlet [5] and Thomée [29]), we also have

Lemma 3.2. There exists a positive constant $C$ such that, for any $v_{h} \in V_{h}$,
(a) $\left\|v_{h}\right\|_{L^{q}} \leq C h^{2 / q-1}\left\|v_{h}\right\|, \quad 2 \leq q \leq \infty$,
(b) $\left\|\nabla v_{h}\right\|_{L^{q}} \leq C h^{2 / q-1}\left\|\nabla v_{h}\right\|, \quad 2 \leq q \leq \infty$,
(c) $\left\|v_{h}\right\|_{1} \leq C h^{-1}\left\|v_{h}\right\|$,
(d) $\left\|v_{h}\right\|_{L^{\infty}} \leq C|\log h|^{1 / 2}\left\|\nabla v_{h}\right\|$. 
Finally, we need (see Ciarlet [5])

Lemma 3.3. There exists a positive constant $C$ such that, for all real $q$ with $1 \leq q \leq \infty$,

(a) $\|\hat{v}\|_{L^{q}(\widehat{K})} \leq C h_{K}^{-2 / q}\|v\|_{L^{q}(K)} \quad \forall v \in L^{q}(K)$,

(b) $\|\nabla \hat{v}\|_{L^{q}(\widehat{K})} \leq C h_{K}^{1-2 / q}\|\nabla v\|_{L^{q}(K)} \quad \forall v \in W_{q}^{1}(K)$,

where $\hat{v}(\zeta)=v(x(\zeta)), x(\zeta)=\sum_{r=j, k, m} x^{r} v^{r}(\zeta)$.

We shall prove the main results of this paper similarly as was done by the author in [31]. By elementary, but tedious computations, we can write the form $\nu_{h}$ as follows:

$$
\begin{gathered}
\nu_{h}\left(\psi_{h} ; n_{h}, v_{h}\right)=a_{1}\left(\psi_{h} ; n_{h}, v_{h}\right)-a_{2}\left(\psi_{h} ; n_{h}, v_{h}\right)-c\left(\psi_{h} ; n_{h}, v_{h}\right) \\
a_{1}\left(\psi_{h} ; n_{h}, v_{h}\right)=\sum_{K \in T_{h}} a^{K}\left\{\alpha_{j}^{K} b_{m k}\left(n_{h m}-n_{h k}\right)\left(v_{h m}-v_{h k}\right)\right. \\
+\alpha_{k}^{K} b_{j m}\left(n_{h j}-n_{h m}\right)\left(v_{h j}-v_{h m}\right) \\
\left.+\alpha_{m}^{K} b_{k j}\left(n_{h k}-n_{h j}\right)\left(v_{h k}-v_{h j}\right)\right\}
\end{gathered}
$$

$$
\begin{aligned}
a_{2}\left(\psi_{h} ; n_{h}, v_{h}\right)=\frac{1}{2} \sum_{K \in T_{h}} b^{K}\left\{b_{m k}\left(n_{h m}-n_{h k}\right)\left(v_{h m}+v_{h k}\right)\right. & \\
+ & b_{j m}\left(n_{h j}-n_{h m}\right)\left(v_{h j}+v_{h m}\right) \\
& \left.+b_{k j}\left(n_{h k}-n_{h j}\right)\left(v_{h k}+v_{h j}\right)\right\}
\end{aligned}
$$

$$
c\left(\psi_{h} ; n_{h}, v_{h}\right)=c_{1}\left(\psi_{h} ; n_{h}, v_{h}\right)-c_{2}\left(\psi_{h} ; n_{h}, v_{h}\right)
$$

$$
\begin{aligned}
& c_{1}\left(\psi_{h} ; n_{h}, v_{h}\right)=\frac{1}{2} \sum_{K \in T_{h}} a^{K}\left\{\alpha_{j}^{K}\left(\psi_{h m}-\psi_{h k}\right)\left(n_{h m}+n_{h k}\right)\left(v_{h m}-v_{h k}\right)\right. \\
& +\alpha_{k}^{K}\left(\psi_{h j}-\psi_{h m}\right)\left(n_{h j}+n_{h m}\right)\left(v_{h j}-v_{h m}\right) \\
& \left.+\alpha_{m}^{K}\left(\psi_{h k}-\psi_{h j}\right)\left(n_{h k}+n_{h j}\right)\left(v_{h k}-v_{h j}\right)\right\} \text {, } \\
& c_{2}\left(\psi_{h} ; n_{h}, v_{h}\right)=\frac{1}{4} \sum_{K \in T_{h}} b^{K}\left\{\left(\psi_{h m}-\psi_{h k}\right)\left(n_{h m}+n_{h k}\right)\left(v_{h m}+v_{h k}\right)\right. \\
& +\left(\psi_{h j}-\psi_{h m}\right)\left(n_{h j}+n_{h m}\right)\left(v_{h j}+v_{h m}\right) \\
& \left.+\left(\psi_{h k}-\psi_{h j}\right)\left(n_{h k}-n_{h j}\right)\left(v_{h k}+v_{h j}\right)\right\} .
\end{aligned}
$$

Here, $\alpha_{r}^{K}=\frac{1}{2} \cot \theta_{r}, r=j, k, m$, where $\theta_{r}$ denotes the measure of the angle of $K$ lying at the vertex $x^{r}$, and $b_{r s}=b_{s r}=\frac{1}{2}\left(B\left(\psi_{h r}-\psi_{h s}\right)+B\left(\psi_{h s}-\psi_{h r}\right)\right)$. From the acuteness and the minimum angle condition it follows immediately that

$$
0 \leq \alpha_{r}^{K} \leq C, \quad r=j, k, m, \forall K \in \bigcup_{h} T_{h}
$$


It is furthermore known that (see Zlámal [34]) $b_{r s} \geq 1$. Therefore,

$$
a_{1}\left(\psi_{h} ; v_{h}, v_{h}\right) \geq a_{1}\left(0 ; v_{h}, v_{h}\right) \geq a_{0}\left\|\nabla v_{h}\right\|^{2} \quad \forall v_{h} \in V_{h}
$$

In a similar way we can derive

$$
\begin{gathered}
\pi_{h}\left(\psi_{h} ; p_{h}, v_{h}\right)=a_{1}\left(\psi_{h} ; p_{h}, v_{h}\right)+a_{2}\left(\psi_{h} ; p_{h}, v_{h}\right)-\bar{c}\left(\psi_{h} ; p_{h}, v_{h}\right) \\
\bar{c}\left(\psi_{h} ; p_{h}, v_{h}\right)=c_{1}\left(\psi_{h} ; p_{h}, v_{h}\right)+c_{2}\left(\psi_{h} ; p_{h}, v_{h}\right) .
\end{gathered}
$$

Theorem 3.1. The problem (2.10) has a unique local solution $\left\{\psi_{h}, n_{h}, p_{h}\right\}$, i.e., there exists a positive constant $T^{*}$ (defined by (3.28)) such that the problem (2.10) is uniquely solvable for $t \in\left[0, T^{*}\right]$.

Proof. (I) Existence. Let $\left\{n_{h}, p_{h}\right\} \in V_{h} \times V_{h}$ be fixed. Then there exists a unique $\psi_{h} \in V_{h}$ such that

$$
d\left(\psi_{h}, v_{h}\right)=\left(p_{h}-n_{h}+N_{I}, v_{h}\right)_{h}, \quad v_{h} \in V_{h} .
$$

Let $v_{h}=\psi_{h}$ in (3.15), and note that

$$
\left\|v_{h}\right\| \leq\left\|v_{h}\right\|_{h} \leq C\left\|v_{h}\right\| \quad \forall v_{h} \in V_{h} .
$$

We obtain

$$
\left\|\nabla \psi_{h}\right\| \leq C\left\{\left\|n_{h}\right\|+\left\|p_{h}\right\|+1\right\}
$$

We denote by $\mathbb{P}$ the mapping from $V_{h}$ into itself, assigning $u_{h}=\left\{n_{h}, p_{h}\right\}$ to $w_{h}=\left\{w_{h}^{(1)}, w_{h}^{(2)}\right\}$ such that

$$
\text { (a) } \begin{aligned}
&\left(\frac{\partial w_{h}^{(1)}}{\partial t}, v_{h}\right)_{h}+a_{1}\left(\psi_{h} ; w_{h}^{(1)}, v_{h}\right) \\
&= a_{2}\left(\psi_{h} ; n_{h}, v_{h}\right)+c\left(\psi_{h} ; n_{h}, v_{h}\right)-\left(R\left(n_{h}, p_{h}\right), v_{h}\right)_{h}, \\
& v_{h} \in V_{h},
\end{aligned}
$$

$$
\begin{aligned}
&\left(\frac{\partial w_{h}^{(2)}}{\partial t}, v_{h}\right)_{h}+a_{1}\left(\psi_{h} ; w_{h}^{(2)}, v_{h}\right) \\
&=-a_{2}\left(\psi_{h} ; p_{h}, v_{h}\right)+\bar{c}\left(\psi_{h} ; p_{h}, v_{h}\right)-\left(R\left(n_{h}, p_{h}\right), v_{h}\right)_{h}, \\
& v_{h} \in V_{h},
\end{aligned}
$$

(c) $w_{h}^{(1)}(0)=n_{I}^{0}, \quad w_{h}^{(2)}(0)=p_{I}^{0}$.

It is easy to see that the problem (3.18) is uniquely solvable. We shall prove the solvability of $(2.10)$ by showing that $\mathbb{P}$ has a fixed point. Let $v_{h}=w_{h}^{(1)}$ in (3.18a), integrate over $\tau$ in $[0, t]$, and note (3.12) and (3.16); the left-hand side of the resulting equation is then bounded below by

$$
\frac{1}{2}\left\{\left\|w_{h}^{(1)}(t)\right\|^{2}-\left\|w_{h}^{(1)}(0)\right\|^{2}\right\}+a_{0} \int_{0}^{t}\left\|\nabla w_{h}^{(1)}(\tau)\right\|^{2} d \tau .
$$


Next, we consider bounds for the terms on the right-hand side. Since (see Zlámal [34])

$$
\left|\chi^{\prime}(\zeta)\right| \leq \frac{1}{2} \quad \forall \zeta \in(-\infty, \infty)
$$

where $\chi(\zeta)=\frac{1}{2}[B(\zeta)+B(-\zeta)]=\frac{1}{2} \zeta\left(e^{\zeta}+1\right) /\left(e^{\zeta}-1\right)$, and $\chi(0)=1$, it follows from (3.7), (3.4), (3.17), and (3.3) that

$$
\begin{aligned}
& \left|\int_{0}^{t} a_{2}\left(\psi_{h} ; n_{h}, w_{h}^{(1)}\right) d \tau\right| \\
& \leq C \int_{0}^{t} \sum_{K \in T_{h}}\left\{\left(1+\frac{1}{2}\left|\psi_{h m}-\psi_{h k}\right|\right)\left|n_{h m}-n_{h k}\right|\left|w_{h m}^{(1)}+w_{h k}^{(1)}\right|+\cdots\right\} d \tau \\
& \leq C \int_{0}^{t} \sum_{K \in T_{h}}\left\{\left\|\nabla \hat{\psi}_{h}\right\|_{L^{2}(\widehat{K})}\left\|\nabla \hat{n}_{h}\right\|_{L^{2}(\widehat{K})}\left\|\hat{w}_{h}^{(1)}\right\|_{L^{\infty}(\widehat{K})}\right. \\
& \left.+\left\|\nabla \hat{n}_{h}\right\|_{L^{2}(\widehat{K})}\left\|\hat{w}_{h}^{(1)}\right\|_{L^{2}(\widehat{K})}\right\} d \tau \\
& \leq C \int_{0}^{t} \sum_{K \in T_{h}}\left\{\left\|\nabla \psi_{h}\right\|_{L^{2}(K)}\left\|\nabla n_{h}\right\|_{L^{2}(K)}\left\|w_{h}^{(1)}\right\|_{L^{\infty}(K)}\right. \\
& \left.+h_{K}^{-1}\left\|\nabla n_{h}\right\|_{L^{2}(K)}\left\|w_{h}^{(1)}\right\|_{L^{2}(K)}\right\} d \tau \\
& \leq C \int_{0}^{t}\left\{\left\|\nabla \psi_{h}\right\|\left\|\nabla n_{h}\right\|\left\|w_{h}^{(1)}\right\|_{L^{\infty}}+h^{-1}\left\|\nabla n_{h}\right\|\left\|w_{h}^{(1)}\right\|\right\} d \tau \\
& \leq C \int_{0}^{t}\left\{h^{-1}|\log h|^{1 / 2}\left(\left\|n_{h}\right\|+\left\|p_{h}\right\|+1\right)\left\|n_{h}\right\|\left\|\nabla w_{h}^{(1)}\right\|\right. \\
& \left.+h^{-2}\left\|n_{h}\right\|\left\|w_{h}^{(1)}\right\|\right\} d \tau \\
& \leq C(h) \int_{0}^{t}\left\{\left(\left\|n_{h}\right\|^{2}+\left\|p_{h}\right\|^{2}\right)^{2}+1\right\} d \tau+\frac{1}{5} a_{0} \int_{0}^{t}\left\|\nabla w_{h}^{(1)}(\tau)\right\|^{2} d \tau \\
& \leq C(h) \int_{0}^{t}\left\{\left\|u_{h}(\tau)\right\|^{4}+1\right\} d \tau+\frac{1}{5} a_{0} \int_{0}^{t}\left\|\nabla w_{h}^{(1)}(\tau)\right\|^{2} d \tau .
\end{aligned}
$$

By (3.9), (3.3), and (3.17),

$$
\begin{aligned}
&\left|\int_{0}^{t} c_{1}\left(\psi_{h} ; n_{h}, w_{h}^{(1)}\right) d \tau\right| \\
& \leq \frac{1}{2} \int_{0}^{t} \sum_{K \in T_{h}} a^{K}\left\{\alpha_{j}^{K}\left|\psi_{h m}-\psi_{h k}\left\|n_{h m}+n_{h k}\right\| w_{h m}^{(1)}-w_{h k}^{(1)}\right|+\cdots\right\} d \tau \\
&(3.22) \quad \leq C \int_{0}^{t}\left\|n_{h}\right\|_{L^{\infty}}\left\|\nabla \psi_{h}\right\|\left\|\nabla w_{h}^{(1)}\right\| d \tau \\
& \quad \leq C h^{-1} \int_{0}^{t}\left\|n_{h}\right\|\left(\left\|n_{h}\right\|+\left\|p_{h}\right\|+1\right)\left\|\nabla w_{h}^{(1)}\right\| d \tau \\
& \quad \leq C(h) \int_{0}^{t}\left\{\left\|u_{h}(\tau)\right\|^{4}+1\right\} d \tau+\frac{1}{5} a_{0} \int_{0}^{t}\left\|\nabla w_{h}^{(1)}(\tau)\right\|^{2} d \tau
\end{aligned}
$$


From (3.10), (3.4), (3.3), and (3.17), we get

$$
\begin{aligned}
& \left|\int_{0}^{t} c_{2}\left(\psi_{h} ; n_{h}, w_{h}^{(1)}\right) d \tau\right| \\
& \quad \leq \frac{1}{4} \int_{0}^{t} \sum_{K \in T_{h}} b^{K}\left\{\left|\psi_{h m}-\psi_{h k}\left\|n_{h m}+n_{h k}\right\| w_{h m}^{(1)}+w_{h k}^{(1)}\right|+\cdots\right\} d \tau \\
& \quad \leq C \int_{0}^{t} \sum_{K \in T_{h}}\left\|\nabla \hat{\psi}_{h}\right\|_{L^{2}(\widehat{K})}\left\|\hat{n}_{h}\right\|_{L^{2}(\widehat{K})}\left\|\hat{w}_{h}^{(1)}\right\|_{L^{\infty}(\widehat{K})} d \tau \\
& \quad \leq C h^{-1} \int_{0}^{t} \sum_{K \in T_{h}}\left\|\nabla \psi_{h}\right\|_{L^{2}(K)}\left\|n_{h}\right\|_{L^{2}(K)}\left\|w_{h}^{(1)}\right\|_{L^{\infty}(K)} d \tau \\
& \quad \leq C h^{-1}|\log h|^{1 / 2} \int_{0}^{t}\left\|\nabla \psi_{h}\right\|\left\|n_{h}\right\|\left\|\nabla w_{h}^{(1)}\right\| d \tau \\
& \quad \leq C(h) \int_{0}^{t}\left\{\left\|u_{h}(\tau)\right\|^{4}+1\right\} d \tau+\frac{1}{5} a_{0} \int_{0}^{t}\left\|\nabla w_{h}^{(1)}(\tau)\right\|^{2} d \tau .
\end{aligned}
$$

Noting that $R(n, p)$ is Lipschitz continuous, and using (3.16), we obtain

$$
\begin{aligned}
& \left|\int_{0}^{t}\left(R\left(n_{h}, p_{h}\right), w_{h}^{(1)}\right)_{h} d \tau\right| \\
& \quad \leq C \int_{0}^{t}\left\{\left\|n_{h}\right\|+\left\|p_{h}\right\|+1\right\}\left\|w_{h}^{(1)}\right\| d \tau \\
& \quad \leq C \int_{0}^{t}\left\{\left\|n_{h}\right\|^{2}+\left\|p_{h}\right\|^{2}+1\right\} d \tau+\frac{1}{5} a_{0} \int_{0}^{t}\left\|\nabla w_{h}^{(1)}(\tau)\right\|^{2} d \tau .
\end{aligned}
$$

Thus, by $(3.18 \mathrm{c})$ and the fact that

$$
\left\|w_{h}^{(1)}(0)\right\| \leq C\left\|n^{0}\right\|,
$$

we have

$$
\begin{aligned}
\left\|w_{h}^{(1)}(t)\right\|^{2} & \leq\left\|w_{h}^{(1)}(0)\right\|^{2}+C(h) \int_{0}^{t}\left\{\left\|u_{h}(\tau)\right\|^{4}+1\right\} d \tau \\
& \leq C\left\|n^{0}\right\|^{2}+C(h) \int_{0}^{t}\left\{\left\|u_{h}(\tau)\right\|^{4}+1\right\} d \tau \\
& \leq C_{1}(h)+C_{1}(h) \int_{0}^{t}\left\{\left\|u_{h}(\tau)\right\|^{4}+1\right\} d \tau .
\end{aligned}
$$

Similarly,

$$
\left\|w_{h}^{(2)}(t)\right\|^{2} \leq C_{2}(h)+C_{2}(h) \int_{0}^{t}\left\{\left\|u_{h}(\tau)\right\|^{4}+1\right\} d \tau .
$$

By (3.25) and (3.26),

$$
\left\|w_{h}(t)\right\|^{2} \leq C^{*}(h)+C^{*}(h) \int_{0}^{t}\left\{\left\|u_{h}(\tau)\right\|^{4}+1\right\} d \tau,
$$

where $C^{*}(h)$ depends on $h,\left\|n^{0}\right\|$, and $\left\|p^{0}\right\|$.

Let

$$
T^{*}=\frac{1}{1+4\left(C^{*}(h)\right)^{2}}>0
$$


and

$$
\mathscr{Q}=\left\{u_{h} \in V_{h} \times V_{h} ;\left\|u_{h}\right\|^{2} \leq 2 C^{*}(h)\right\} .
$$

Then, by (3.27), for $0 \leq t \leq T^{*}$, we can prove that $\mathbb{P}(\mathscr{Q}) \subset \mathscr{Q}$. By usual arguments one can show that $\mathbb{P}$ is continuous. Therefore, the Brouwer Fixed Point Theorem yields the existence of a fixed point of $\mathbb{P}$.

(II) Uniqueness. Let $\left\{\psi_{h}, n_{h}, p_{h}\right\}$ and $\left\{\bar{\psi}_{h}, \bar{n}_{h}, \bar{p}_{h}\right\}$ be solutions of (2.10) for $t \in\left[0, T^{*}\right]$, and let $\tilde{\psi}=\psi_{h}-\bar{\psi}_{h}, \tilde{n}=n_{h}-\bar{n}_{h}$, and $\tilde{p}=p_{h}-\bar{p}_{h}$. Then

$$
\|\nabla \tilde{\psi}\| \leq C\{\|\tilde{n}\|+\|\tilde{p}\|\}
$$

and

$$
\begin{aligned}
\left(\frac{\partial \tilde{n}}{\partial t},\right. & \left.v_{h}\right)_{h}+a_{1}\left(\psi_{h} ; \tilde{n}, v_{h}\right) \\
= & a_{1}\left(\bar{\psi}_{h} ; \bar{n}_{h}, v_{h}\right)-a_{1}\left(\psi_{h} ; \bar{n}_{h}, v_{h}\right) \\
& +a_{2}\left(\bar{\psi}_{h} ; \bar{n}_{h}, v_{h}\right)-a_{2}\left(\psi_{h} ; n_{h}, v_{h}\right)+c\left(\bar{\psi}_{h} ; \bar{n}_{h}, v_{h}\right) \\
& -c\left(\psi_{h} ; n_{h}, v_{h}\right)-\left(R\left(n_{h}, p_{h}\right)-R\left(\bar{n}_{h}, \bar{p}_{h}\right), v_{h}\right)_{h}, \quad v_{h} \in V_{h} .
\end{aligned}
$$

Let $v_{h}=\tilde{n}$ in (3.31); then, by (3.12),

$$
\begin{aligned}
\frac{1}{2} \frac{d}{d t}\|\tilde{n}\|_{h}^{2}+a_{0}\|\nabla \tilde{n}\|^{2} \leq & \left|a_{1}\left(\bar{\psi}_{h} ; \bar{n}_{h}, \tilde{n}\right)-a_{1}\left(\psi_{h} ; \bar{n}_{h}, \tilde{n}\right)\right| \\
& +\left|a_{2}\left(\bar{\psi}_{h} ; \bar{n}_{h}, \tilde{n}\right)-a_{2}\left(\psi_{h} ; n_{h}, \tilde{n}\right)\right| \\
& +\left|c\left(\bar{\psi}_{h} ; \bar{n}_{h}, \tilde{n}\right)-c\left(\psi_{h} ; n_{h}, \tilde{n}\right)\right| \\
& +\left|\left(R\left(n_{h}, p_{h}\right)-R\left(\bar{n}_{h}, \bar{p}_{h}\right), \tilde{n}\right)_{h}\right| \\
= & I_{1}+I_{2}+I_{3}+I_{4} .
\end{aligned}
$$

We integrate (3.32) over $\tau$ in $I_{t}=[0, t]$ for $t \in I^{*}=\left[0, T^{*}\right]$ and note that $\tilde{n}(0)=0$ and (3.16); then the left-hand side of the resulting equation is bounded below by

$$
\frac{1}{2}\|\tilde{n}(t)\|^{2}+a_{0} \int_{0}^{t}\|\nabla \tilde{n}(\tau)\|^{2} d \tau .
$$

On the right-hand side of the resulting equation, we have, by (3.6), (3.20), (3.3), (3.30), and $\left\|\bar{n}_{h}\right\|_{L^{\infty}\left(I^{*} ; L^{2}\right)} \leq C(h)$, that

$$
\begin{aligned}
\int_{0}^{t} I_{1} d \tau & \leq \int_{0}^{t} \sum_{K \in T_{h}} a^{K}\left\{\alpha_{j}^{K}\left|b_{m k}-\bar{b}_{m k}\left\|\bar{n}_{h m}-\bar{n}_{h k}\right\| \tilde{n}_{m}-\tilde{n}_{k}\right|+\cdots\right\} d \tau \\
& \leq C \int_{0}^{t} \sum_{K \in T_{h}}\left\{\left|\tilde{\psi}_{m}-\tilde{\psi}_{k}\left\|\bar{n}_{h m}-\bar{n}_{n k}\right\| \tilde{n}_{m}-\tilde{n}_{k}\right|+\cdots\right\} d \tau \\
& \leq C \int_{0}^{t}\|\nabla \tilde{\psi}\|\left\|\bar{n}_{h}\right\|_{L^{\infty}}\|\nabla \tilde{n}\| d \tau \\
& \leq C h^{-1} \int_{0}^{t}\|\nabla \tilde{\psi}\|\left\|\bar{n}_{h}\right\|\|\nabla \tilde{n}\| d \tau \\
& \leq C(h) \int_{0}^{t}\left\{\|\tilde{n}\|^{2}+\|\tilde{p}\|^{2}\right\} d \tau+\frac{a_{0}}{5} \int_{0}^{t}\|\nabla \tilde{n}\|^{2} d \tau .
\end{aligned}
$$

Noting (3.7), (3.20), (3.3), (3.30), and

$$
\left\|\nabla \bar{\psi}_{h}\right\|_{L^{\infty}\left(I^{*} ; L^{2}\right)}+\left\|n_{h}\right\|_{L^{\infty}\left(I^{*} ; L^{2}\right)} \leq C(h),
$$


50

JIANG ZHU

we get

$$
\begin{aligned}
& \int_{0}^{t} I_{2} d \tau \leq \int_{0}^{t}\left\{\left|a_{2}\left(\bar{\psi}_{h} ; \bar{n}_{h}, \tilde{n}\right)-a_{2}\left(\bar{\psi}_{h} ; n_{h}, \tilde{n}\right)\right|\right. \\
& \left.+\left|a_{2}\left(\bar{\psi}_{h} ; n_{h}, \tilde{n}\right)-a_{2}\left(\psi_{h} ; n_{h}, \tilde{n}\right)\right|\right\} d \tau \\
& \leq C \int_{0}^{t} \sum_{K \in T_{h}}\left\{\left[\left|\bar{\psi}_{h m}-\bar{\psi}_{h k}\left\|\tilde{n}_{m}-\tilde{n}_{k}\right\| \tilde{n}_{m}+\tilde{n}_{k}\right|+\cdots\right]\right. \\
& +\left[\left|\tilde{n}_{m}-\tilde{n}_{k}\right|\left|\tilde{n}_{m}+\tilde{n}_{k}\right|+\cdots\right] \\
& \left.\left.+\left|\tilde{\psi}_{h m}-\tilde{\psi}_{h k}\right|\left|n_{h m}-n_{h k}\right|\left|\tilde{n}_{m}+\tilde{n}_{k}\right|+\cdots\right]\right\} d \tau \\
& \leq C \int_{0}^{t}\left\{\left\|\nabla \bar{\psi}_{h}\right\|\|\nabla \tilde{n}\|\|\tilde{n}\|_{L^{\infty}}+h^{-1}\|\tilde{n}\|\|\nabla \tilde{n}\|\right. \\
& \left.+\|\nabla \tilde{\psi}\|\left\|\nabla n_{h}\right\|\|\tilde{n}\|_{L^{\infty}}\right\} d \tau \\
& \leq C h^{-1} \int_{0}^{t}\left\{\left\|\nabla \bar{\psi}_{h}\right\|\|\nabla \tilde{n}\|\|\tilde{n}\|+\|\tilde{n}\|\|\nabla \tilde{n}\|\right. \\
& \left.+|\log h|^{1 / 2}\|\nabla \tilde{\psi}\|\left\|n_{h}\right\|\|\nabla \tilde{n}\|\right\} d \tau \\
& \leq C(h) \int_{0}^{t}\left\{\|\tilde{n}\|^{2}+\|\tilde{p}\|^{2}\right\} d \tau+\frac{a_{0}}{5} \int_{0}^{t}\|\nabla \tilde{n}\|^{2} d \tau \text {. }
\end{aligned}
$$

Breaking $I_{3}$ into two parts, we have

$$
\begin{aligned}
I_{3} & \leq\left|c\left(\bar{\psi}_{h} ; \bar{n}_{h}, \tilde{n}\right)-c\left(\bar{\psi}_{h} ; n_{h}, \tilde{n}\right)\right|+\left|c\left(\bar{\psi}_{h} ; n_{h}, \tilde{n}\right)-c\left(\psi_{h} ; n_{h}, \tilde{n}\right)\right| \\
& =\left|c\left(\bar{\psi}_{h} ; \tilde{n}, \tilde{n}\right)\right|+\left|c\left(\tilde{\psi} ; n_{h}, \tilde{n}\right)\right|=I_{31}+I_{32} .
\end{aligned}
$$

From (3.8)-(3.10), (3.4), (3.3), and (3.35), we obtain

$$
\begin{aligned}
\int_{0}^{t} I_{31} d \tau & \leq C \int_{0}^{t} \sum_{K \in T_{h}}\left\{\left[\left|\bar{\psi}_{h m}-\bar{\psi}_{h k}\left\|\tilde{n}_{m}+\tilde{n}_{k}\right\| \tilde{n}_{m}-\tilde{n}_{k}\right|+\cdots\right]\right. \\
& \left.\quad+\left[\left|\bar{\psi}_{h m}-\bar{\psi}_{h k} \| \tilde{n}_{m}+\tilde{n}_{k}\right|^{2}+\cdots\right]\right\} d \tau \\
& \leq C \int_{0}^{t}\left\{\left\|\nabla \bar{\psi}_{h}\right\|\|\nabla \tilde{n}\|\|\tilde{n}\|_{L^{\infty}}+h^{-1}\left\|\nabla \bar{\psi}_{h}\right\|\|\tilde{n}\|\|\tilde{n}\|_{L^{\infty}}\right\} d \tau \\
& \leq C(h) \int_{0}^{t}\left\|\nabla \bar{\psi}_{h}\right\|\|\nabla \tilde{n}\|\|\tilde{n}\| d \tau \\
& \leq C(h) \int_{0}^{t}\|\tilde{n}\|^{2} d \tau+\frac{a_{0}}{5} \int_{0}^{t}\|\nabla \tilde{n}\|^{2} d \tau .
\end{aligned}
$$

By noting (3.30), we get

$$
\begin{aligned}
\int_{0}^{t} I_{32} d \tau \leq & \leq C \int_{0}^{t} \sum_{K \in T_{h}}\left\{\left[\left|\tilde{\psi}_{m}-\tilde{\psi}_{k}\left\|n_{h m}+n_{h k}\right\| \tilde{n}_{m}-\tilde{n}_{k}\right|+\cdots\right]\right. \\
& \left.\quad+\left[\left|\tilde{\psi}_{m}-\tilde{\psi}_{k}\left\|n_{h m}+n_{h k}\right\| \tilde{n}_{m}+\tilde{n}_{k}\right|+\cdots\right]\right\} d \tau \\
& \leq C \int_{0}^{t}\left\{\|\nabla \tilde{\psi}\|\|\nabla \tilde{n}\|\left\|n_{h}\right\|_{L^{\infty}}+h^{-1}\|\nabla \tilde{\psi}\|\|\tilde{n}\|\left\|n_{h}\right\|_{L^{\infty}}\right\} d \tau \\
& \leq C(h) \int_{0}^{t}\|\nabla \tilde{\psi}\|\|\nabla \tilde{n}\|\left\|n_{h}\right\| d \tau \\
& \leq C(h) \int_{0}^{t}\left\{\|\tilde{n}\|^{2}+\|\tilde{p}\|^{2}\right\} d \tau+\frac{a_{0}}{5} \int_{0}^{t}\|\nabla \tilde{n}\|^{2} d \tau .
\end{aligned}
$$


Noting that $R(n, p)$ is Lipschitz continuous, and using (3.16), we easily see that

$$
\int_{0}^{t} I_{4} d \tau \leq C \int_{0}^{t}\left\{\|\tilde{n}\|^{2}+\|\tilde{p}\|^{2}\right\} d \tau .
$$

From (3.32)-(3.34) and (3.36)-(3.40), we have, for each $t \in I^{*}$,

$$
\|\tilde{n}(t)\|^{2} \leq C(h) \int_{0}^{t}\left\{\|\tilde{n}\|^{2}+\|\tilde{p}\|^{2}\right\} d \tau .
$$

Similarly,

$$
\|\tilde{p}(t)\|^{2} \leq C(h) \int_{0}^{t}\left\{\|\tilde{n}\|^{2}+\|\tilde{p}\|^{2}\right\} d \tau .
$$

By (3.41) and (3.42),

$$
\|\tilde{n}(t)\|^{2}+\|\tilde{p}(t)\|^{2} \leq C(h) \int_{0}^{t}\left\{\|\tilde{n}\|^{2}+\|\tilde{p}\|^{2}\right\} d \tau .
$$

Gronwall's Lemma and (3.30) now complete the proof.

Let $e_{\psi}=\psi-\psi_{h}, e_{n}=n-n_{h}=n-\bar{n}+\bar{n}-n_{h}=\eta_{n}+\xi_{n}$, and $e_{p}=p-p_{h}=$ $p-\bar{p}+\bar{p}-p_{h}=\eta_{p}+\xi_{p}$. Then we have

Theorem 3.2. With $T^{*}$ defined by (3.28), there exists a positive constant $C$ such that, for $h$ sufficiently small

$$
\begin{aligned}
& \left\|\xi_{n}\right\|_{L^{\infty}\left(I^{*} ; L^{2}\right)}+\left\|\xi_{p}\right\|_{L^{\infty}\left(I^{*} ; L^{2}\right)} \\
& \quad+\left\|\nabla \xi_{n}\right\|_{L^{2}\left(I^{*} ; L^{2}\right)}+\left\|\nabla \xi_{p}\right\|_{L^{2}\left(I^{*} ; L^{2}\right)} \leq C h^{\sigma} .
\end{aligned}
$$

Proof. Subtract (2.10a) from (2.4a) to obtain

$$
\begin{aligned}
d\left(e_{\psi}, v_{h}\right)= & \left(p-n+N, v_{h}\right)-\left(p_{h}-n_{h}+N_{I}, v_{h}\right)_{h} \\
= & \left(e_{p}-e_{n}+N-N_{I}, v_{h}\right)+\left(p_{h}-n_{h}+N_{I}, v_{h}\right) \\
& -\left(p_{h}-n_{h}+N_{I}, v_{h}\right)_{h}, \quad v_{h} \in V_{h} .
\end{aligned}
$$

From Lemma 4.3 in Zlámal [34], (2.6), and (3.2), we have, for each $v_{h} \in V_{h}$,

$$
\begin{aligned}
d\left(e_{\psi}, v_{h}\right) \leq & \left\{\left\|e_{n}\right\|+\left\|e_{p}\right\|+\left\|N-N_{I}\right\|\right\}\left\|v_{h}\right\|+C h\left\{\left\|n_{h}\right\|+\left\|p_{h}\right\|+\left\|N_{I}\right\|\right\}\left\|\nabla v_{h}\right\| \\
\leq & \left\{\left\|e_{n}\right\|+\left\|e_{p}\right\|+\left\|N-N_{I}\right\|\right\}\left\|v_{h}\right\| \\
& +C h\left\{1+\left\|e_{n}\right\|+\left\|e_{p}\right\|+\left\|N-N_{I}\right\|\right\}\left\|\nabla v_{h}\right\| \\
\leq & \left\{\left\|\xi_{n}\right\|+\left\|\xi_{p}\right\|+\left\|\eta_{n}\right\|+\left\|\eta_{p}\right\|+\left\|N-N_{I}\right\|\right\}\left\|v_{h}\right\| \\
& +C h\left\{1+\left\|\xi_{n}\right\|+\left\|\xi_{p}\right\|+\left\|\eta_{n}\right\|+\left\|\eta_{p}\right\|+\left\|N-N_{I}\right\|\right\}\left\|\nabla v_{h}\right\| \\
\leq & \left\{\left\|\xi_{n}\right\|+\left\|\xi_{p}\right\|+h\right\}\left\|v_{h}\right\|+C h\left\{1+\left\|\xi_{n}\right\|+\left\|\xi_{p}\right\|+h\right\}\left\|\nabla v_{h}\right\| .
\end{aligned}
$$

Thus, by $(3.2 \mathrm{e})$,

$$
\begin{aligned}
\left\|\nabla e_{\psi}\right\|^{2} & =d\left(e_{\psi}, e_{\psi}\right)=d\left(e_{\psi}, \psi-\psi_{I}\right)+d\left(e_{\psi}, \psi_{I}-\psi_{h}\right) \\
& \leq \frac{1}{2}\left\|\nabla e_{\psi}\right\|^{2}+C\left\{\left\|\nabla\left(\psi-\psi_{I}\right)\right\|^{2}+\left\|\xi_{n}\right\|^{2}+\left\|\xi_{p}\right\|^{2}+h^{2}\right\} \\
& \leq \frac{1}{2}\left\|\nabla e_{\psi}\right\|^{2}+C\left\{\left\|\xi_{n}\right\|^{2}+\left\|\xi_{p}\right\|^{2}+h^{2 \sigma}\right\} .
\end{aligned}
$$


Therefore,

$$
\left\|\nabla e_{\psi}\right\|^{2} \leq C\left\{\left\|\xi_{n}\right\|^{2}+\left\|\xi_{p}\right\|^{2}+h^{2 \sigma}\right\}
$$

To estimate $\xi_{n}$, subtract $(2.10 \mathrm{~b})$ from $(2.4 \mathrm{~b})$. Letting

$$
\begin{gathered}
A_{1}^{K}(n, v)=\left(A_{1}^{K} \nabla n, \nabla v\right), \\
\nu^{K}(\psi ; n, v)=A_{1}^{K}(n, v)-\left(n A_{1}^{K} \nabla \psi, \nabla v\right),
\end{gathered}
$$

and noting $(2.5),(3.1),(3.5)$, and $a_{1}\left(0 ; \bar{n}, v_{h}\right)-a_{2}\left(0 ; \bar{n}, v_{h}\right)=A_{1}^{K}\left(\bar{n}, v_{h}\right)$ (obtained by (2.7), (3.5)-(3.10), (3.45)), we have, for each $v_{h} \in V_{h}$,

$$
\begin{aligned}
\left(\frac{d \xi_{n}}{\partial t},\right. & \left.v_{h}\right)_{h}+a_{1}\left(\psi_{h} ; \xi_{h}, v_{h}\right) \\
= & \left(\frac{\partial \bar{n}}{\partial t}, v_{h}\right)_{h}-\left(\frac{\partial \bar{n}}{\partial t}, v_{h}\right)+\nu^{K}\left(\psi ; n, v_{h}\right)-\nu\left(\psi ; n, v_{h}\right) \\
& +a_{1}\left(\psi_{h} ; \bar{n}, v_{h}\right)-a_{1}\left(0 ; \bar{n}, v_{h}\right)-\left\{a_{2}\left(\psi_{h} ; n_{h}, v_{h}\right)-a_{2}\left(0 ; \bar{n}, v_{h}\right)\right\} \\
& +\left(n A_{1}^{K} \nabla \psi, \nabla v_{h}\right)-\left(n_{h} A_{1}^{K} \nabla \psi_{h}, \nabla v_{h}\right) \\
& +\left(n_{h} A_{1}^{K} \nabla \psi_{h}, \nabla v_{h}\right)-c\left(\psi_{h} ; n_{h}, v_{h}\right) \\
& -A_{1}^{K}\left(\eta_{n}, v_{h}\right)+\left(R\left(n_{h}, p_{h}\right), v_{h}\right)_{h}-\left(R(n, p), v_{h}\right) .
\end{aligned}
$$

Let $v_{h}=\xi_{n}$ in $(3.47)$, and note that

$$
a_{2}\left(0 ; \xi_{n}, \xi_{n}\right)=\frac{1}{2} \sum_{K \in T_{h}} b^{K}\left[\left(\xi_{n m}^{2}-\xi_{n k}^{2}\right)+\left(\xi_{n j}^{2}-\xi_{n m}^{2}\right)+\left(\xi_{n k}^{2}-\xi_{n j}^{2}\right)\right]=0
$$

so, $a_{2}\left(0 ; \bar{n}, \xi_{n}\right)=a_{2}\left(0 ; n_{h}, \xi_{n}\right)$; then by $(3.12)$, we have

$$
\begin{aligned}
\frac{1}{2} \frac{d}{d t}\left\|\xi_{n}\right\|_{h}^{2}+a_{0}\left\|\nabla \xi_{n}\right\|^{2} \\
\leq\left|\left(\frac{\partial \bar{n}}{\partial t}, \xi_{n}\right)_{h}-\left(\frac{\partial \bar{n}}{\partial t}, \xi_{n}\right)\right|+\left|\nu^{K}\left(\psi ; n, \xi_{n}\right)-\nu\left(\psi ; n, \xi_{n}\right)\right| \\
\quad+\left|a_{1}\left(\psi_{h} ; \bar{n}, \xi_{n}\right)-a_{1}\left(0 ; \bar{n}, \xi_{n}\right)\right|+\left|a_{2}\left(\psi_{h} ; n_{h}, \xi_{n}\right)-a_{2}\left(0 ; n_{h}, \xi_{n}\right)\right| \\
\quad+\left|\left(n A_{1}^{K} \nabla \psi, \nabla \xi_{n}\right)-\left(n_{h} A_{1}^{K} \nabla \psi_{h}, \nabla \xi_{n}\right)\right| \\
\quad+\left|\left(n_{h} A_{1}^{K} \nabla \psi_{h}, \nabla \xi_{n}\right)-c\left(\psi_{h} ; n_{h}, \xi_{n}\right)\right| \\
\\
+\left|A_{1}^{K}\left(\eta_{n}, \xi_{n}\right)\right|+\left|\left(R\left(n_{h}, p_{h}\right), \xi_{n}\right)_{h}-\left(R(n, p), \xi_{n}\right)\right|=\sum_{i=1}^{8} F_{i} .
\end{aligned}
$$

Integrating (3.48) termwise over $\tau$ in $I_{t}=[0, t]$ for $t \in I^{*}$, we find that the left-hand side of the resulting equation is bounded below by

$$
\frac{1}{2}\left\{\left\|\xi_{n}(t)\right\|_{h}^{2}-\left\|\xi_{n}(0)\right\|_{h}^{2}\right\}+a_{0}\left\|\nabla \xi_{n}\right\|_{L^{2}\left(I_{t} ; L^{2}\right)}^{2}
$$


Next, we consider bounds for the terms on the right-hand side of the resulting equation. Using Lemma 4.3 in Zlámal [34], (3.1b) and (2.6c), we have

$$
\int_{0}^{t} F_{1} d \tau \leq C h \int_{0}^{t}\left\|\frac{\partial \bar{n}}{\partial t}\right\|\left\|\nabla \xi_{n}\right\| d \tau \leq \frac{a_{0}}{16}\left\|\nabla \xi_{n}\right\|_{L^{2}\left(I_{t} ; L^{2}\right)}^{2}+C h^{2} .
$$

By (2.5), (3.45), and (3.46), and noting that $\beta(x)$ is Lipschitz continuous, and (2.6), we get

$$
\begin{aligned}
\int_{0}^{t} F_{2} d \tau & \leq \int_{0}^{t}\left\{\sum_{K \in T_{h}} \int_{K}\left|A_{1}-A_{1}^{K}\|\nabla n-n \nabla \psi\| \nabla \xi_{n}\right| d x\right\} d \tau \\
& \leq C h \int_{0}^{t} \sum_{K \in T_{h}}\|\nabla n-n \nabla \psi\|_{L^{2}(K)}\left\|\nabla \xi_{n}\right\|_{L^{2}(K)} d \tau \\
& \leq C h \int_{0}^{t}\|\nabla n-n \nabla \psi\|\left\|\nabla \xi_{n}\right\| d \tau \\
& \leq C h \int_{0}^{t}\left\{\|\nabla n\|+\|\nabla \psi\|\|n\|_{L^{\infty}}\right\}\left\|\nabla \xi_{n}\right\| d \tau \\
& \leq \frac{a_{0}}{16}\left\|\nabla \xi_{n}\right\|_{L^{2}\left(I_{t} ; L^{2}\right)}^{2}+C h^{2} .
\end{aligned}
$$

From (3.6), (3.20), (3.4), (3.3), the Sobolev Imbedding Theorem, (3.2), (3.44), and (2.6), we deduce

$$
\begin{aligned}
\int_{0}^{t} F_{3} d \tau & \leq \int_{0}^{t} \sum_{K \in T_{h}} a^{K}\left\{\alpha_{j}^{K}\left|b_{m k}-1\left\|\bar{n}_{m}-\bar{n}_{k}\right\| \xi_{n m}-\xi_{n k}\right|+\cdots\right\} d \tau \\
& \leq C \int_{0}^{t} \sum_{K \in T_{h}}\left\{\left|\psi_{h m}-\psi_{h k}\right|\left[\left|n_{m}-n_{k}\right|+\left|\eta_{n m}-\eta_{n k}\right|\right]\right. \\
& \left.\times C \xi_{n m}-\xi_{n k} \mid+\cdots\right\} d \tau \\
& =\sum_{K \in T_{h}}\left\{\left\|\nabla \hat{\psi}_{h}\right\|_{L^{2}(\widehat{K})}\left\|\nabla \hat{n}_{I}\right\|_{L^{2 /(1-\sigma)}(\widehat{K})}\left\|\nabla \hat{\xi}_{n}\right\|_{L^{2 / \sigma}(\widehat{K})}\right. \\
& +C \int_{0}^{t} \sum_{K \in T_{h}} h_{K}\left\{\left\|\nabla \psi_{h}\right\|_{L^{2}(K)}\left\|\nabla n_{I}\right\|_{L^{2 /(1-\sigma)}(K)}\left\|\nabla \xi_{n}\right\|_{L^{2 / \sigma}(K)}\right. \\
& +C h \int_{0}^{t}\left\|\nabla \psi_{h}\right\|\left\{\|\nabla n\|_{L^{2 /(1-\sigma)}}\left\|\nabla \xi_{n}\right\|_{L^{2 / \sigma}}+\left\|\nabla \eta_{n}\right\|\left\|\nabla \xi_{n}\right\|_{L^{\infty}}\right\} d \tau \\
& \leq C \int_{0}^{t}\left\{\|\nabla \psi\|+\left\|\nabla e_{\psi}\right\|\right\}\left\{h^{\sigma}\|\nabla n\|_{L^{2 /(1-\sigma)}}+\left\|\nabla \eta_{n}\right\|\right\}\left\|\nabla \xi_{n}\right\| d \tau \\
& \leq C h^{\sigma} \int_{0}^{t}\left\{\|\psi\|_{1+\sigma}+\left\|\nabla \hat{\xi}_{\psi}\right\|\right\}\|n\|_{1+\sigma}\left\|\nabla \xi_{n}\right\| d \tau \\
& \leq \frac{a_{0}}{16}\left\|\nabla \xi_{n}\right\|_{L^{2}\left(I_{t} ; L^{2}\right)}^{2}+C h^{2 \sigma}\left\{\left\|\xi_{n}\right\|_{L^{\infty}\left(I_{t} ; L^{2}\right)}^{2}+\left\|\xi_{p}\right\|_{L^{\infty}\left(I_{t} ; L^{2}\right)}^{2}+1\right\}
\end{aligned}
$$


Breaking $F_{4}$ into pieces, we have

$$
\begin{gathered}
F_{4} \leq \frac{1}{2} \sum_{K \in T_{h}} b^{K}\left\{\left|b_{m k}-1\right|\left|n_{h m}-n_{h k}\right|\left|\xi_{n m}+\xi_{n k}\right|+\cdots\right\} \\
\leq C \sum_{K \in T_{h}}\left\{\left|\psi_{h m}-\psi_{h k} \| n_{h m}-n_{h k}\right|\left|\xi_{n m}+\xi_{n k}\right|+\cdots\right\} \\
\leq C \sum_{K \in T_{h}}\left\{\left[\left|\psi_{m}-\psi_{k}\right|\left|n_{m}-n_{k}\right|\left|\xi_{n m}+\xi_{n k}\right|+\cdots\right]\right. \\
+\left[\left|\psi_{m}-\psi_{k} \| \xi_{n m}-\xi_{n k}\right|\left|\xi_{n m}+\xi_{n k}\right|+\cdots\right] \\
+\left[\left|\psi_{m}-\psi_{k} \| \eta_{n m}-\eta_{n k}\right|\left|\xi_{n m}+\xi_{n k}\right|+\cdots\right] \\
+\left[\left|e_{\psi m}-e_{\psi k} \| n_{m}-n_{k}\right|\left|\xi_{n m}+\xi_{n k}\right|+\cdots\right] \\
+\left[\left|e_{\psi m}-e_{\psi k}\right|\left|\xi_{n m}-\xi_{n k}\right|\left|\xi_{n m}+\xi_{n k}\right|+\cdots\right] \\
\left.+\left[\left|e_{\psi m}-e_{\psi k} \| \eta_{n m}-\eta_{n k}\right|\left|\xi_{n m}+\xi_{n k}\right|+\cdots\right]\right\}
\end{gathered}
$$

By (3.4), the Sobolev Imbedding Theorem, (3.3), and (2.6), we get

$$
\begin{aligned}
\int_{0}^{t} F_{41} d \tau & \leq C \int_{0}^{t} \sum_{K \in T_{h}}\left\{\left|\psi_{m}-\psi_{k}\left\|n_{m}-n_{k}\right\| \xi_{n m}+\xi_{n k}\right|+\cdots\right\} d \tau \\
& \leq C \int_{0}^{t} \sum_{K \in T_{h}}\left\|\nabla \hat{\psi}_{I}\right\|_{L^{2 /(1-\sigma)}(\widehat{K})}\left\|\nabla \hat{n}_{I}\right\|_{L^{2 /(1-\sigma)}(\widehat{K})}\left\|\hat{\xi}_{n}\right\|_{L^{1 / \sigma}(\widehat{K})} d \tau \\
& \leq C \int_{0}^{t} \sum_{K \in T_{h}}\left\|\nabla \hat{\psi}_{I}\right\|_{L^{2 /(1-\sigma)}(\widehat{K})}\left\|\nabla \hat{n}_{I}\right\|_{L^{2 /(1-\sigma)}(\widehat{K})}\left\|\hat{\xi}_{n}\right\|_{L^{\infty}(\widehat{K})} d \tau \\
& \leq C h^{2 \sigma} \int_{0}^{t} \sum_{K \in T_{h}}\left\|\nabla \psi_{I}\right\|_{L^{2 /(1-\sigma)}(K)}\left\|\nabla n_{I}\right\|_{L^{2 /(1-\sigma)}(K)}\left\|\xi_{n}\right\|_{L^{\infty}(K)} d \tau \\
& \leq C h^{2 \sigma} \int_{0}^{t} \sum_{K \in T_{h}}\|\psi\|_{H^{1+\sigma}(K)}\|n\|_{H^{1+\sigma}(K)}\left\|\xi_{n}\right\|_{L^{\infty}(K)} d \tau \\
& \leq C h^{2 \sigma} \int_{0}^{t}\|\psi\|_{1+\sigma}\|n\|_{1+\sigma}\left\|\xi_{n}\right\|_{L^{\infty}} d \tau \\
& \leq C h^{2 \sigma}|\log h|^{1 / 2} \int_{0}^{t}\|\psi\|_{1+\sigma}\|n\|_{1+\sigma}\left\|\nabla \xi_{n}\right\| d \tau \\
& \leq \frac{a_{0}}{16}\left\|\nabla \xi_{n}\right\|_{L^{2}\left(I_{t} ; L^{2}\right)}^{2}+C h^{4 \sigma}|\log h|
\end{aligned}
$$


Using the Gagliardo-Nirenberg Inequality

$$
\|v\|_{L^{q}} \leq C(q)\|v\|^{2 / q}\|\nabla v\|^{(q-2) / q}, \quad v \in V, q>2,
$$

and Young's Inequality

$$
a b \leq C(\delta, q) a^{q}+\delta b^{q /(1-q)}, \quad a, b \geq 0, q>1,
$$

we have (let $q=2 / \sigma)$

$$
\begin{aligned}
\int_{0}^{t} F_{42} d \tau & \leq C \int_{0}^{t} \sum_{K \in T_{h}}\left\{\left|\psi_{m}-\psi_{k}\left\|\xi_{n m}-\xi_{n k}\right\| \xi_{n m}+\xi_{n k}\right|+\cdots\right\} d \tau \\
& \leq C \int_{0}^{t} \sum_{K \in T_{h}}\left\|\nabla \hat{\psi}_{I}\right\|_{L^{2 /(1-\sigma)}(\widehat{K})}\left\|\nabla \hat{\xi}_{n}\right\|_{L^{2}(\widehat{K})}\left\|\hat{\xi}_{n}\right\|_{L^{2 / \sigma}(\widehat{K})} d \tau \\
& \leq C \int_{0}^{t} \sum_{K \in T_{h}}\left\|\nabla \psi_{I}\right\|_{L^{2 /(1-\sigma)}(K)}\left\|\nabla \xi_{n}\right\|_{L^{2}(K)}\left\|\xi_{n}\right\|_{L^{2 / \sigma}(K)} d \tau \\
& \leq C \int_{0}^{t}\|\nabla \psi\|_{\left.L^{2 /(1-\sigma)}\right)}\left\|\nabla \xi_{n}\right\|\left\|\xi_{n}\right\|_{L^{2 / \sigma}} d \tau \\
& \leq C \int_{0}^{t}\|\psi\|_{1+\sigma}\left\|\xi_{n}\right\|^{\sigma}\left\|\nabla \xi_{n}\right\|^{2-\sigma} d \tau \\
& \leq \frac{a_{0}}{16}\left\|\nabla \xi_{n}\right\|_{L^{2}\left(I_{t} ; L^{2}\right)}^{2}+C\left\|\xi_{n}\right\|_{L^{2}\left(I_{t} ; L^{2}\right)}^{2}
\end{aligned}
$$

and by (3.4), (3.2), and (3.3),

$$
\begin{aligned}
\int_{0}^{t} F_{43} d \tau & \leq C \int_{0}^{t} \sum_{K \in T_{h}}\left\{\left|\psi_{m}-\psi_{k}\left\|\eta_{n m}-\eta_{n k}\right\| \xi_{n m}+\xi_{n k}\right|+\cdots\right\} d \tau \\
& \leq C \int_{0}^{t} \sum_{K \in T_{h}}\left\|\nabla \hat{\psi}_{I}\right\|_{L^{2 /(1-\sigma)}(\widehat{K})}\left\|\nabla\left(\hat{\eta}_{n}\right)_{I}\right\|_{L^{2}(\widehat{K})}\left\|\hat{\xi}_{n}\right\|_{L^{2 / \sigma}(\widehat{K})} d \tau \\
& \leq C \int_{0}^{t} \sum_{K \in T_{h}}\left\|\nabla \psi_{I}\right\|_{L^{2 /(1-\sigma)}(K)}\left\|\nabla\left(\eta_{n}\right)_{I}\right\|_{L^{2}(K)}\left\|\xi_{n}\right\|_{L^{2 / \sigma}(K)} d \tau \\
& \leq C \int_{0}^{t}\|\nabla \psi\|_{L^{2 /(1-\sigma)} \|}\left\|\nabla \eta_{n}\right\|\left\|\xi_{n}\right\|_{L^{2 / \sigma}} d \tau \\
& \leq C \int_{0}^{t}\|\psi\|_{1+\sigma}\left\|\nabla \eta_{n}\right\|\left\|\nabla \xi_{n}\right\| d \tau \\
& \leq \frac{a_{0}}{16}\left\|\nabla \xi_{n}\right\|_{L^{2}\left(I_{t} ; L^{2}\right)}^{2}+C h^{2 \sigma} .
\end{aligned}
$$


From (3.4), the Sobolev Imbedding Theorem, (3.3), (2.6), and (3.44), we get

$$
\begin{aligned}
\int_{0}^{t} F_{44} d \tau \leq & C \int_{0}^{t} \sum_{K \in T_{h}}\left\{\left|e_{\psi m}-e_{\psi k}\left\|n_{m}-n_{k}\right\| \xi_{n m}+\xi_{n k}\right|+\cdots\right\} d \tau \\
\leq & C \int_{0}^{t} \sum_{K \in T_{h}}\left\|\nabla\left(\hat{e}_{\psi}\right)_{I}\right\|_{L^{2}(\widehat{K})}\left\|\nabla \hat{n}_{I}\right\|_{L^{2 /(1-\sigma)}(\widehat{K})}\left\|\hat{\xi}_{n}\right\|_{L^{2 / \sigma}(\widehat{K})} d \tau \\
\leq & C \int_{0}^{t} \sum_{K \in T_{h}}\left\|\nabla\left(\hat{e}_{\psi}\right)_{I}\right\|_{L^{2}(\widehat{K})}\left\|\nabla \hat{n}_{I}\right\|_{L^{2 /(1-\sigma)}(\widehat{K})}\left\|\hat{\xi}_{n}\right\|_{L^{\infty}(\widehat{K})} d \tau \\
\leq & C h^{\sigma} \int_{0}^{t} \sum_{K \in T_{h}}\left\|\nabla\left(e_{\psi}\right)_{I}\right\|_{L^{2}(K)}\left\|\nabla n_{I}\right\|_{L^{2 /(1-\sigma)}(K)}\left\|\xi_{n}\right\|_{L^{\infty}(K)} d \tau \\
\leq & C h^{\sigma} \int_{0}^{t} \sum_{K \in T_{h}}\left\|\nabla e_{\psi}\right\|_{L^{2}(K)}\|n\|_{H^{1+\sigma}(K)}\left\|\xi_{n}\right\|_{L^{\infty}(K)} d \tau \\
\leq & C h^{\sigma}|\log h|^{1 / 2} \int_{0}^{t}\left\|\nabla e_{\psi}\right\|\|n\|_{1+\sigma}\left\|\nabla \xi_{n}\right\| d \tau \\
\leq & \frac{a_{0}}{16}\left\|\nabla \xi_{n}\right\|_{L^{2}\left(I_{t} ; L^{2}\right)}^{2} \\
& +C h^{2 \sigma}|\log h|\left\{\left\|\xi_{n}\right\|_{L^{\infty}\left(I_{t} ; L^{2}\right)}^{2}+\left\|\xi_{p}\right\|_{L^{\infty}\left(I_{t} ; L^{2}\right)}^{2}+h^{2 \sigma}\right\}
\end{aligned}
$$

$\int_{0}^{t} F_{45} d \tau \leq C \int_{0}^{t} \sum_{K \in T_{h}}\left\{\left|e_{\psi m}-e_{\psi k}\left\|\xi_{n m}-\xi_{n k}\right\| \xi_{n m}+\xi_{n k}\right|+\cdots\right\} d \tau$

$$
\leq C \int_{0}^{t} \sum_{K \in T_{h}}\left\|\nabla\left(\hat{e}_{\psi}\right)_{I}\right\|_{L^{2}(\widehat{K})}\left\|\nabla \hat{\xi}_{n}\right\|_{L^{2}(\widehat{K})}\left\|\hat{\xi}_{n}\right\|_{L^{\infty}(\widehat{K})} d \tau
$$

$$
\begin{aligned}
& \leq C \int_{0}^{t} \sum_{K \in T_{h}}\left\|\nabla\left(e_{\psi}\right)_{I}\right\|_{L^{2}(K)}\left\|\nabla \xi_{n}\right\|_{L^{2}(K)}\left\|\xi_{n}\right\|_{L^{\infty}(K)} d \tau \\
& \leq C \int_{0}^{t}\left\|\nabla e_{\psi}\right\|\left\|\nabla \xi_{n}\right\|\left\|\xi_{n}\right\|_{L^{\infty}} d \tau \\
& \leq C|\log h|^{1 / 2}\left\{\left\|\xi_{n}\right\|_{L^{\infty}\left(I_{t} ; L^{2}\right)}+\left\|\xi_{p}\right\|_{L^{\infty}\left(I_{t} ; L^{2}\right)}+h^{\sigma}\right\}\left\|\nabla \xi_{n}\right\|_{L^{2}\left(I_{t} ; L^{2}\right)}^{2}, \\
& \int_{0}^{t} F_{46} d \tau \leq C \int_{0}^{t} \sum_{K \in T_{h}}\left\{\left|e_{\psi m}-e_{\psi k}\left\|\eta_{n m}-\eta_{n k}\right\| \xi_{n m}+\xi_{n k}\right|+\cdots\right\} d \tau \\
& \leq C \int_{0}^{t} \sum_{K \in T_{h}}\left\|\nabla\left(\hat{e}_{\psi}\right)_{I}\right\|_{L^{2}(\widehat{K})}\left\|\nabla\left(\hat{\eta}_{n}\right)_{I}\right\|_{L^{2}(\widehat{K})}\left\|\hat{\xi}_{n}\right\|_{L^{\infty}(\widehat{K})} d \tau \\
& \leq C \int_{0}^{t} \sum_{K \in T_{h}}\left\|\nabla\left(e_{\psi}\right)_{I}\right\|_{L^{2}(K)}\left\|\nabla\left(\eta_{n}\right)_{I}\right\|_{L^{2}(K)}\left\|\xi_{n}\right\|_{L^{\infty}(K)} d \tau \\
& \leq C \int_{0}^{t}\left\|\nabla e_{\psi}\right\|\left\|\nabla \eta_{n}\right\|\left\|\xi_{n}\right\|_{L^{\infty}} d \tau \\
& \leq \frac{a_{0}}{16}\left\|\nabla \xi_{n}\right\|_{L^{2}\left(I_{t} ; L^{2}\right)}^{2} \\
&+C h^{2 \sigma}|\log h|\left\{\left\|\xi_{n}\right\|_{L^{\infty}\left(I_{t} ; L^{2}\right)}^{2}+\left\|\xi_{p^{\prime}}\right\|_{L^{\infty}\left(I_{t} ; L^{2}\right)}^{2}+h^{2 \sigma}\right\} .
\end{aligned}
$$


In order to estimate $F_{5}$, we shall break it into pieces as follows:

$$
\begin{aligned}
F_{5}= & \left|\left(A_{1}^{K} e_{n} \nabla \psi, \nabla \xi_{n}\right)\right|+\left|\left(A_{1}^{K} \bar{n} \nabla e_{\psi}, \nabla \xi_{n}\right)\right|+\left|\left(A_{1}^{K} \xi_{n} \nabla e_{\psi}, \nabla \xi_{n}\right)\right| \\
= & \left|\left(A_{1}^{K} \xi_{n} \nabla \psi, \nabla \xi_{n}\right)\right|+\left|\left(A_{1}^{K} \eta_{n} \nabla \psi, \nabla \xi_{n}\right)\right| \\
& +\left|\left(A_{1}^{K} \bar{n} \nabla e_{\psi}, \nabla \xi_{n}\right)\right|+\left|\left(A_{1}^{K} \xi_{n} \nabla e_{\psi}, \nabla \xi_{n}\right)\right| \\
= & F_{51}+F_{52}+F_{53}+F_{54} .
\end{aligned}
$$

From Remark 2.1, (2.6), and using the Sobolev Imbedding Theorem, the Gagliardo-Nirenberg Inequality (3.55), and Young's Inequality (3.56), we have

$$
\begin{aligned}
\int_{0}^{t} F_{51} d \tau & \leq M \int_{0}^{t}\left\|\xi_{n}\right\|_{L^{2 / \sigma}}\|\nabla \psi\|_{L^{2 /(1-\sigma)}}\left\|\nabla \xi_{n}\right\|_{L^{2}} d \tau \\
& \leq C\|\psi\|_{L^{\infty}\left(H^{1+\sigma}\right)} \int_{0}^{t}\left\|\xi_{n}\right\|^{\sigma}\left\|\nabla \xi_{n}\right\|^{2-\sigma} d \tau \\
& \leq \frac{a_{0}}{16}\left\|\nabla \xi_{n}\right\|_{L^{2}\left(I_{l} ; L^{2}\right)}^{2}+C\left\|\xi_{n}\right\|_{L^{2}\left(I_{t} ; L^{2}\right)}^{2} .
\end{aligned}
$$

Furthermore, by (3.2),

$$
\begin{aligned}
\int_{0}^{t} F_{52} d \tau & \leq M \int_{0}^{t}\left\|\eta_{n}\right\|_{L^{2 / \sigma}}\|\nabla \psi\|_{L^{2 /(1-\sigma)}}\left\|\nabla \xi_{n}\right\|_{L^{2}} d \tau \\
& \leq C\|\psi\|_{L^{\infty}\left(H^{1+\sigma}\right)} \int_{0}^{t}\left\|\nabla \eta_{n}\right\|\left\|\nabla \xi_{n}\right\| d \tau \\
& \leq \frac{a_{0}}{16}\left\|\nabla \xi_{n}\right\|_{L^{2}\left(I_{t} ; L^{2}\right)}^{2}+C h^{2 \sigma}
\end{aligned}
$$

by $(3.44)$,

$$
\begin{aligned}
\int_{0}^{t} F_{53} d \tau & \leq M \int_{0}^{t}\|\bar{n}\|_{L^{\infty}}\left\|\nabla e_{\psi}\right\|\left\|\nabla \xi_{n}\right\| d \tau \\
& \leq C\|n\|_{L^{\infty}\left(L^{\infty}\right)} \int_{0}^{t}\left\|\nabla e_{\psi}\right\|\left\|\nabla \xi_{n}\right\| d \tau \\
& \leq \frac{a_{0}}{16}\left\|\nabla \xi_{n}\right\|_{L^{2}\left(I_{t} ; L^{2}\right)}^{2}+C\left\|\nabla e_{\psi}\right\|_{L^{2}\left(I_{t} ; L^{2}\right)}^{2} \\
& \leq \frac{a_{0}}{16}\left\|\nabla \xi_{n}\right\|_{L^{2}\left(I_{t} ; L^{2}\right)}^{2}+C\left\{\left\|\xi_{n}\right\|_{L^{2}\left(I_{t} ; L^{2}\right)}^{2}+\left\|\xi_{p}\right\|_{L^{2}\left(I_{t} ; L^{2}\right)}^{2}+h^{2 \sigma}\right\}
\end{aligned}
$$

and by (3.3),

$$
\begin{aligned}
\int_{0}^{t} F_{54} d \tau & \leq M \int_{0}^{t}\left\|\xi_{n}\right\|_{L^{\infty}}\left\|\nabla e_{\psi}\right\|\left\|\nabla \xi_{n}\right\| d \tau \\
\leq C|\log h|^{1 / 2}\left\{\left\|\xi_{n}\right\|_{L^{\infty}\left(I_{t} ; L^{2}\right)}\right. & \\
& \left.+\left\|\xi_{p}\right\|_{L^{\infty}\left(I_{t} ; L^{2}\right)}+h^{\sigma}\right\}\left\|\nabla \xi_{n}\right\|_{L^{2}\left(I_{t} ; L^{2}\right)}^{2}
\end{aligned}
$$

Since

$$
A_{i}^{K}\left(\psi_{n}, \xi_{h}\right)=\sum_{K \in T_{h}} \int_{K} A_{1}^{K} \nabla \psi_{h} \cdot \nabla \xi_{h} d x=\sum_{K \in T_{h}} \operatorname{area}(K) A_{1}^{K} \nabla \psi_{h} \cdot \nabla \xi_{h}
$$

and

$$
\begin{aligned}
A_{1}^{K}\left(\psi_{h}, \xi_{h}\right)=\nu_{h}\left(0 ; \psi_{h}, \xi_{h}\right)= & \sum_{K \in T_{h}} a^{K}\left[\alpha_{j}^{K}\left(\psi_{h m}-\psi_{h k}\right)\left(\xi_{n m}-\xi_{n k}\right)+\cdots\right] \\
& -\frac{1}{2} \sum_{K \in T_{h}} b^{K}\left[\left(\psi_{h m}-\psi_{h k}\right)\left(\xi_{n m}+\xi_{n k}\right)+\cdots\right],
\end{aligned}
$$


we get

$$
\begin{aligned}
\operatorname{area}(K) A_{1}^{K} \nabla \psi_{h} \cdot \nabla \xi_{h}= & a^{K}\left[\alpha_{j}^{K}\left(\psi_{h m}-\psi_{h k}\right)\left(\xi_{n m}-\xi_{n k}\right)+\cdots\right] \\
& -\frac{1}{2} b^{K}\left[\left(\psi_{h m}-\psi_{h k}\right)\left(\xi_{n m}+\xi_{n k}\right)+\cdots\right]
\end{aligned}
$$

and

$$
\begin{aligned}
& F_{6}=\left|c\left(\psi_{h} ; n_{h}, \xi_{n}\right)-\sum_{K \in T_{h}} 2 \operatorname{area}(K) A_{1}^{K} \nabla \psi_{h} \cdot \nabla \xi_{h} \int_{\widehat{K}} \hat{n}_{h} d \zeta\right| \\
&= \mid \sum_{K \in T_{h}} a^{K}\left\{\alpha_{j}^{K}\left(\psi_{h m}-\psi_{h k}\right)\left(\xi_{n m}-\xi_{n k}\right)\left[\frac{1}{2}\left(n_{h m}+n_{h k}\right)-2 \int_{\widehat{K}} \hat{n}_{h} d \zeta\right]+\cdots\right\} \\
&-\frac{1}{2} \sum_{K \in T_{h}} b^{K}\left\{\left(\psi_{h m}-\psi_{h k}\right)\left(\xi_{n m}+\xi_{n k}\right)\right. \\
&=\left.\left.\times \mid \frac{1}{2}\left(n_{h m}+n_{h k}\right)-2 \int_{\widehat{K}} \hat{n}_{h} d \zeta\right]+\cdots\right\} \mid \\
& \quad-\frac{1}{2} \sum_{K \in T_{h}} a^{K}\left\{\alpha_{j}^{K}\left(\psi_{h m}-\psi_{h k}\right)\left(\xi_{n m}-\xi_{n k}\right)\left[\left(n_{h m}-n_{h j}\right)+\left(n_{h k}-n_{h j}\right)\right]+\cdots\right\} \\
& \leq C \sum_{K \in T_{h}}\left\{\left|\psi_{h m}-\psi_{h k}\right|\left[\left|\xi_{n m}-\xi_{n k}\right|+\left|\xi_{n m}+\xi_{n k}\right|\right]\right. \\
&\left.\times\left|\left(n_{h m}-n_{h j}\right)+\left(n_{h k}-n_{h j}\right)\right|+\cdots\right\} .
\end{aligned}
$$

Similarly to the treatment of $F_{4}$, we can show that

$$
\begin{aligned}
\int_{0}^{t} F_{6} d \tau \leq & \frac{a_{0}}{16}\left\|\nabla \xi_{n}\right\|_{L^{2}\left(I_{t} ; L^{2}\right)}^{2}+C\left\|\xi_{n}\right\|_{L^{2}\left(I_{t} ; L^{2}\right)}^{2} \\
& +C h^{2 \sigma}|\log h|\left\{\left\|\xi_{n}\right\|_{L^{\infty}\left(I_{t} ; L^{2}\right)}^{2}+\left\|\xi_{p}\right\|_{L^{\infty}\left(I_{t} ; L^{2}\right)}^{2}+h^{2 \sigma}\right\} \\
& +C|\log h|^{1 / 2}\left\{\left\|\xi_{n}\right\|_{L^{\infty}\left(I_{t} ; L^{2}\right)}+\left\|\xi_{p}\right\|_{L^{\infty}\left(I_{t} ; L^{2}\right)}+h^{\sigma}\right\}\left\|\nabla \xi_{n}\right\|_{L^{2}\left(I_{t} ; L^{2}\right)}^{2}
\end{aligned}
$$

By Remark 2.1 and (3.2),

$$
\int_{0}^{t} F_{7} d \tau \leq M \int_{0}^{t}\left\|\nabla \eta_{n}\right\|\left\|\nabla \xi_{n}\right\| d \tau \leq \frac{a_{0}}{16}\left\|\nabla \xi_{n}\right\|_{L^{2}\left(I_{t} ; L^{2}\right)}^{2}+C h^{2 \sigma} .
$$

Using Theorem 4.1.5 in Ciarlet [5], we have

$$
\begin{aligned}
& \left|\left(R(n, p), \xi_{n}\right)_{h}-\left(R(n, p), \xi_{n}\right)\right| \\
& \quad \leq C \sum_{K \in T_{h}} h_{K}(\operatorname{area}(K))^{\sigma / 2}\|R(n, p)\|_{W_{2 /(1-\sigma)}^{1}(K)}\left\|\nabla \xi_{n}\right\|_{L^{2}(K)} \\
& \quad \leq C h(\operatorname{area}(\Omega))^{\sigma / 2}\|R(n, p)\|_{W_{2 /(1-\sigma)}^{1}}\left\|\nabla \xi_{n}\right\| \\
& \quad \leq C h\left\{\|n\|_{1+\sigma}+\|p\|_{1+\sigma}+1\right\}\left\|\nabla \xi_{n}\right\|
\end{aligned}
$$


where, in the last inequality, we have made use of (3.13) in Zlámal [33] and the Sobolev Imbedding Theorem. Hence, by (3.16), (3.2), and (2.6),

$$
\begin{aligned}
\int_{0}^{t} F_{8} d \tau \leq & \int_{0}^{t}\left\{\left|\left(R(n, p), \xi_{n}\right)_{h}-\left(R(n, p), \xi_{n}\right)\right|\right. \\
& \left.+\left|\left(R\left(n_{h}, p_{h}\right), \xi_{n}\right)_{h}-\left(R(n, p), \xi_{n}\right)_{h}\right|\right\} d \tau \\
\leq & \frac{a_{0}}{16}\left\|\nabla \xi_{n}\right\|_{L^{2}\left(I_{t} ; L^{2}\right)}^{2}+C\left\{\left\|\xi_{n}\right\|_{L^{2}\left(I_{t} ; L^{2}\right)}^{2}+\left\|\xi_{p}\right\|_{L^{2}\left(I_{t} ; L^{2}\right)}^{2}+h^{2 \sigma}\right\} .
\end{aligned}
$$

Combining (3.48)-(3.54), (3.57)-(3.69), and noting (3.16), $\left\|\xi_{n}(0)\right\| \leq$ $\left\|n^{0}-n_{I}^{0}\right\|+\left\|n^{0}-\bar{n}^{0}\right\| \leq C h^{\sigma}$, and $C|\log h|^{1 / 2} h^{\sigma} \leq a_{0} / 16$ for $h$ sufficiently small, we have

$$
\begin{aligned}
\frac{1}{2} \| \xi_{n}(t) & \left\|^{2}+\frac{a_{0}}{16}\right\| \nabla \xi_{n} \|_{L^{2}\left(I_{t} ; L^{2}\right)}^{2} \\
\leq & C|\log h|^{1 / 2}\left\{\left\|\xi_{n}\right\|_{L^{\infty}\left(I_{t} ; L^{2}\right)}+\left\|\xi_{p}\right\|_{L^{\infty}\left(I_{t} ; L^{2}\right)}\right\}\left\|\nabla \xi_{n}\right\|_{L^{2}\left(I_{t} ; L^{2}\right)}^{2} \\
& +C\left\{\left\|\xi_{n}\right\|_{L^{2}\left(I_{t} ; L^{2}\right)}^{2}+\left\|\xi_{p}\right\|_{L^{2}\left(I_{t} ; L^{2}\right)}^{2}+h^{2 \sigma}\right\} \\
& +C h^{2 \sigma}|\log h|\left\{\left\|\xi_{n}\right\|_{L^{\infty}\left(I_{t} ; L^{2}\right)}^{2}+\left\|\xi_{p}\right\|_{L^{\infty}\left(I_{t} ; L^{2}\right)}^{2}\right\}
\end{aligned}
$$

Similarly, we have

$$
\begin{aligned}
\frac{1}{2} \| \xi_{p}(t) & \left\|^{2}+\frac{a_{0}}{16}\right\| \nabla \xi_{p} \|_{L^{2}\left(I_{t} ; L^{2}\right)}^{2} \\
\leq & C|\log h|^{1 / 2}\left\{\left\|\xi_{n}\right\|_{L^{\infty}\left(I_{t} ; L^{2}\right)}+\left\|\xi_{p}\right\|_{L^{\infty}\left(I_{t} ; L^{2}\right)}\right\}\left\|\nabla \xi_{p}\right\|_{L^{2}\left(I_{t} ; L^{2}\right)}^{2} \\
& +C\left\{\left\|\xi_{n}\right\|_{L^{2}\left(I_{t} ; L^{2}\right)}^{2}+\left\|\xi_{p}\right\|_{L^{2}\left(I_{t} ; L^{2}\right)}^{2}+h^{2 \sigma}\right\} \\
& +C h^{2 \sigma}|\log h|\left\{\left\|\xi_{n}\right\|_{L^{\infty}\left(I_{t} ; L^{2}\right)}^{2}+\left\|\xi_{p}\right\|_{L^{\infty}\left(I_{t} ; L^{2}\right)}^{2}\right\}
\end{aligned}
$$

Let $\xi=\left\{\xi_{n}, \xi_{p}\right\} ;$ then

$$
\begin{aligned}
\frac{1}{2} \| \xi(t) & \left\|^{2}+\frac{a_{0}}{16}\right\| \nabla \xi \|_{L^{2}\left(I_{t} ; L^{2}\right)}^{2} \\
\leq & C|\log h|^{1 / 2}\|\xi\|_{L^{\infty}\left(I_{t} ; L^{2}\right)}\|\nabla \xi\|_{L^{2}\left(I_{t} ; L^{2}\right)}^{2} \\
& +C\left\{\|\xi\|_{L^{2}\left(I_{t} ; L^{2}\right)}^{2}+h^{2 \sigma}\right\}+C h^{2 \sigma}|\log h|\|\xi\|_{L^{\infty}\left(I_{t} ; L^{2}\right)}^{2}
\end{aligned}
$$

As in $[6,11,31]$, let us make the induction hypothesis that

$$
C|\log h|^{1 / 2}\|\xi\|_{L^{\infty}\left(I^{*} ; L^{2}\right)} \leq \frac{a_{0}}{32} .
$$

Obviously, (3.73) holds for $t=0$. Thus, (3.73) will hold for $t \leq t^{*}$ for some $t^{*}>0$. We shall show for $h$ sufficiently small that $t^{*}=T^{*}$ and that (3.43) holds.

It follows from (3.72), (3.73), and Gronwall's Lemma that

$$
\|\xi\|_{L^{\infty}\left(I^{*} ; L^{2}\right)}^{2}+\|\nabla \xi\|_{L^{2}\left(I^{*} ; L^{2}\right)}^{2} \leq C h^{2 \sigma},
$$

where $C$ is independent of $T^{*}$. Note that (3.74) implies that the induction hypothesis (3.73) holds for small $h$, so that the entire argument is validated. 
From Theorem 3.2 and (3.2), one easily obtains the following corollary.

Corollary 3.1. Let

$$
\widetilde{M}=\left\{\|n\|_{L^{\infty}\left(L^{2}\right)}+\|p\|_{L^{\infty}\left(L^{2}\right)}\right\}+1 ;
$$

then

$$
\left\|n_{h}\right\|_{L^{\infty}\left(I^{*} ; L^{2}\right)}+\left\|p_{h}\right\|_{L^{\infty}\left(I^{*} ; L^{2}\right)} \leq \widetilde{M} .
$$

If we substitute $\widetilde{M}$ defined by (3.75) for $\left\|n^{0}\right\|_{L^{2}}$ and $\left\|p^{0}\right\|_{L^{2}}$ on which $C^{*}(h)$ (in (3.27)) depends, then $T^{*}$ defined by (3.28) depends only on $h$. Thus, for $h$ fixed, $T^{*}$ is a fixed constant, and we can show that the problem (2.10) has a unique global solution by extending gradually the local solution defined by Theorem 3.1. Therefore, Theorem 3.2 holds for $I$ instead of $I^{*}$. Noting (3.2) and (3.44), we have the following main results of this paper.

Theorem 3.3. Problem (2.10) is uniquely solvable. Let $\{\psi, n, p\}$ satisfy (1.5)(1.8) and $\left\{\psi_{h}, n_{h}, p_{h}\right\}$ satisfy (2.10). If the regularity assumptions (2.6) hold, then there exists a positive constant $C$ such that, for $h$ sufficiently small,

$$
\begin{gathered}
\left\|n-n_{h}\right\|_{L^{\infty}\left(L^{2}\right)}+\left\|p-p_{h}\right\|_{L^{\infty}\left(L^{2}\right)}+\left\|\nabla\left(\psi-\psi_{h}\right)\right\|_{L^{\infty}\left(L^{2}\right)} \\
+\left\|\nabla\left(n-n_{h}\right)\right\|_{L^{2}\left(L^{2}\right)}+\left\|\nabla\left(p-p_{h}\right)\right\|_{L^{2}\left(L^{2}\right)} \leq C h^{\sigma} .
\end{gathered}
$$

Corollary 3.2. Theorem 3.3 holds for the case when $\vec{B}=0$, considered by Zlámal [33, 34].

\section{ACKNOWLEDGMENT}

The author thanks Professor Yuanming Wang for introducing this subject to him and for some helpful discussions. He is also grateful to the referee for very valuable comments and suggestions. The work was partly supported by the National Natural Science Foundation of China.

\section{BIBLIOGRAPHY}

1. W. Allegretto, Y. S. Mun, A. Nathan, and H. P. Baltes, Optimization of semiconductor magnetic field sensors using finite element analysis, Proc. NASECODE IV Conf., Boole Press, Dublin, 1985, pp. 129-133.

2. J. Banasiak and G. F. Roach, On mixed boundary value problems of Dirichlet obliquederivative type in plane domains with piecewise differentiable boundary, J. Differential Equations 79 (1989), 111-131.

3. R. E. Bank, W. M. Fichtner, Jr., D. J. Rose, and R. K. Smith, Transient simulation of silicon devices and circuits, IEEE Trans. Computer-Aided Design 4 (1985), 436-451.

4. R. E. Bank, J. W. Jerome, and D. J. Rose, Analytical and numerical aspects of semiconductor device modeling, Proc. Fifth Internat. Conf. on Computing Methods in Applied Science and Engineering (R. Glowinski and J. L. Lions, eds.), North-Holland, Amsterdam, 1982, pp. 593-597.

5. P. G. Ciarlet, The finite element method for elliptic problems, North-Holland, Amsterdam, New York, Oxford, 1978.

6. J. Douglas, Jr., R. E. Ewing, and M. F. Wheeler, The approximation of the pressure by $a$ mixed method in the simulation of miscible displacement, RAIRO Anal. Numer. 17 (1983), 17-34. 
7. J. Douglas, Jr., I. M. Gamba, and M. C. J. Squeff, Simulation of the transient behavior of a one-dimensional semiconductor device, Mat. Apl. Comput. 5 (1986), 103-122.

8. J. Douglas, Jr. and Yirang Yuan, Finite difference methods for the transient behavior of a semiconductor device, Mat. Apl. Comput. 6 (1987), 25-38.

9. J. Douglas, Jr., Yirang Yuan, and Gang Li, A modified method of characteristic procedure for the transient behavior of a semiconductor device (preprint).

10. __ A mixed method for the transient behavior of a semiconductor device (preprint).

11. R. E. Ewing, Time-stepping Galerkin methods for nonlinear Sobolev partial differential equations, SIAM J. Numer. Anal. 15 (1978), 1125-1150.

12. R. E. Ewing and M. F. Wheeler, Galerkin methods for miscible displacement problems with point sources and sinks-unit mobility ratio case, Lectures on the Numerical Solution of Partial Differential Equations, University of Maryland, 1981, pp. 151-174.

13. H. Gajewski, On existence, uniqueness and asymptotic behavior of solutions of the basic equations for carrier transport in semiconductors, Z. Angew. Math. Mech. 65 (1985), 101108.

14. H. Gajewski and K. Gröger, On the basic equations for carrier transport in semiconductors, J. Math. Anal. Appl. 113 (1986), 12-35.

15. I. M. Gamba and M. C. J. Squeff, Simulation of the transient behavior of a one-dimensional semiconductor device. II, SIAM J. Numer. Anal. 26 (1989), 539-552.

16. V. Girault and P.-A. Raviart, Finite element methods for Navier-Stokes equations: Theory and algorithms, Springer Ser. Comput. Math., vol. 5, Springer-Verlag, Berlin, Heidelberg, New York, Tokyo, 1986.

17. J. W. Jerome, Consistency of semiconductor modeling. An existence/stability analysis for the stationary Van Roosbroeck system, SIAM J. Appl. Math. 45 (1985), 565-590.

18. __ Evolution systems in semiconductor modeling. A cyclic uncoupled analysis for the Gummel map (to appear).

19. P. A. Markowich, $A$ singular perturbation analysis of the fundamental semiconductor device equations, SIAM J. Appl. Math. 44 (1984), 896-928.

20. __ The stationary semiconductor device equations, Springer-Verlag, Wien-New York, 1985.

21. P. A. Markowich and C. Ringhofer, A singularly perturbed boundary value problem modelling a semiconductor device, SIAM J. Appl. Math. 44 (1984), 231-256.

22. P. A. Markowich and M. A. Zlámal, Inverse-average-type finite element discretizations of selfadjoint second-order elliptic problems, Math. Comp. 51 (1988), 431-449.

23. M. S. Mock, On equations describing steady-state carrier distributions in a semiconductor device, Comm. Pure Appl. Math. 25 (1972), 781-792.

24. _ An initial value problem from semiconductor device theory, SIAM J. Math. Anal. 5 (1974), 597-612.

25. __ Analysis of mathematical models of semiconductor devices, Boole Press, Dublin, 1983.

26. C. Ringhofer and C. Schmeiser, An approximate Newton method for the solution of the basic semiconductor device equations, SIAM J. Numer. Anal. 26 (1989), 507-516.

27. S. Selberherr and C. Ringhofer, Discretization methods for the semiconductor equations, Proc. NASECODE III Conf., Boole Press, Dublin, 1983, pp. 31-45.

28. E. Stephan and J. R. Whiteman, Singularities of the Laplacian at corners and edges of threedimensional domains and their treatment with finite element methods, Math. Methods Appl. Sci. 10 (1988), 339-350.

29. V. Thomée, Galerkin finite element methods for parabolic problems, Lecture Notes in Math., vol. 1054, Springer-Verlag, 1984.

30. Yuanming Wang, Mathematical model and its analysis for the carrier transport in semiconductor devices, Appl. Math. J. Chinese Univ. 2 (1987), 228-240.

31. Jiang Zhu, The finite element methods for nonlinear Sobolev equation, Northeast. Math. J. 5 (1989), 179-196. 
32. Jiang Zhu, Finite difference methods for the semiconductor device equations with magnetic influence (to appear).

33. M. A. Zlámal, Finite element solution of the fundamental equations of semiconductor devices. I, Math. Comp. 46 (1986), 27-43.

34. _ Finite element solution of the fundamental equations of semiconductor devices. II (to appear).

Department of Mathematics and Mechanics, Southeast University, Nanjing 210018, People's Republic OF China 